Submitted to the Astrophysical Journal

\title{
THE COSMOLOGICAL DENSITY AND IONIZATION OF HOT GAS: OXYGEN VI ABSORPTION IN QUASAR SPECTRA ${ }^{1}$
}

\author{
SCOTT BURLES and DAVID TYTLER ${ }^{2}$ \\ Department of Physics, and Center for Astrophysics and Space Sciences \\ University of California, San Diego \\ C0111, La Jolla, CA 92093-0111
}

\begin{abstract}
We have conducted the first survey for Oxygen VI $\lambda \lambda 1032,1038$ absorption lines in QSO spectra. We used medium resolution $(R \approx 1300)$ high signal-to-noise $(\approx 20)$ Faint Object Spectrograph spectra of 11 QSOs $\left(0.53 \leq z_{e m} \leq 2.08\right)$ from the Hubble Space Telescope Archive. We use simulated spectra to determine the significance of the line identifications, which lie exclusively in the Ly $\alpha$ forest.

We found $12 \mathrm{O}$ VI doublets of which 9 are expected to be real and 6 constitute a uniform sample with both lines exceeding a rest equivalent width of $W_{r}=0.21 \AA$. The number of O VI doublets per unit redshift at a mean absorption redshift of $z_{\text {ave }}=0.9$ is $\langle N(z)\rangle=1.0 \pm 0.6$, which is similar to the density of C IV and Mg II absorbers.

In 7 of the $12 \mathrm{O}$ VI systems, O VI, Ly $\beta$ and $\mathrm{C}$ IV lines have similar equivalent widths, and are probably photoionized. In each of the remaining 5 systems, O VI has larger equivalent widths than those detected for $\operatorname{Ly} \beta$ and $\mathrm{C} I V$. These systems are labeled as high ionization and are likely to be due to collisional ionization. These would be the first QSO absorption systems known to be collisionally ionized.

Assuming that the O VI lines are on the linear part of the curve of growth, we estimate the lower limit of the cosmological mass density, $\Omega(O V I) \geq 1 \times 10^{-8} h_{100}^{-1}$. Since O $>$ O VI, if the mean cosmic metallicity, Z, were below $6 \times 10^{-4}$ solar, then the accompanying Hydrogen and Helium would account for all baryons in the universe. We conclude that $\log Z(z=0.9) / Z_{\odot} \geq-3.2$, and much greater if $\mathrm{O}$ VI is not the dominant ion of Oxygen.
\end{abstract}

Subject headings: cosmology - galaxy: intergalactic medium - galaxy: abundance quasars: absorption lines

\footnotetext{
${ }^{1}$ Based on observations obtained with the NASA/ESA Hubble Space Telescope obtained by the Space Telescope Science Institute, which is operated by AURA, Inc., under NASA contract NAS5-26555.

${ }^{2}$ scott@cass154.ucsd.edu,tytler@cass155.ucsd.edu
} 


\section{INTRODUCTION}

We are interested in O VI absorption in QSO spectra because it is the easiest way to find cosmologically distributed hot gas with $T \approx 3 \times 10^{5} \mathrm{~K}$. Such gas may have been missed from existing surveys of QSO absorption systems which require damped Ly $\alpha$ lines, Lyman continuum absorption, and Mg II or C IV lines. Unlike other QSO absorption systems, this hot gas might be collisionally ionized and could comprise the bulk of all baryons.

\subsection{Source of Ionization}

We are extremely interested in whether the O VI gas is photoionized or collisionally ionized. We consider collisional ionization because gas of the required temperatures exists, and there may be insufficient high energy photons for photoionization, especially at low redshifts $(z \leq 1)$. We are also interested in the source of the ionizing energy - mechanical heating of the gas, or high energy UV photons.

Gas at temperatures needed to collisionally ionize O VI has been seen in galaxies, so we expect to see it in QSO absorption spectra. Pettini \& D'Odorico (1986) have detected Fe X absorption from million degree gas in the Galactic halo and the Large Magellanic Cloud, while Davidsen et al. (1991) have seen O VI in absorption from gas in the our Galaxy's halo along the direction to $3 \mathrm{C} 273$. High latitude clouds in our Galaxy produce shadows in the soft X-ray background (Burrows \& Mendenhall 1991, Snowden et al. 1991) which reveal gas at $T \sim 10^{6} \mathrm{~K}$

in the halo of our Galaxy, and similar halos have been detected in other late-type galaxies (Wang 1991). But the X-ray halos of early-type galaxies are too hot $-5-23 \times 10^{6} \mathrm{~K}-$ to show strong metal lines in the HST wavelength range (Forman et al. 1979, Fabbiano 1989).

Photoionization is known to the dominate in most QSO absorption systems which show strong C IV or Mg II (Bergeron 1988), but O VI is different. Photons with energies above $114 \mathrm{eV}$ are needed to create $\mathrm{O}$ VI, and these are scarce because they are absorbed by both Hydrogen and Helium. QSOs and AGN are the likely sources of such photons. QSOs and AGN apparently do emit at these energies because they show strong O VI emission, and Ne VIII ( $>207 \mathrm{eV})$ emission lines are also common (Hamann, Zuo \& Tytler 1995). But we do not know if this radiation escapes from the QSO environment, or from the host galaxies, which might be opaque in the He II continuum.

The He II Gunn-Peterson absorption provides a measure of the spectrum of the intergalactic background radiation field. The IGM He II/H I ratio depends on the flux of photons capable of ionizing He II to He III, and H I to H II. Jakobsen et al. (1994) find that the optical depth at He II $304 \AA$ is $\tau(304) \geq 1.7$, which indicates that the spectrum of the radiation which ionizes the IGM is very steep. Madua (1994) detects little flux above 4 Rydberg, therefore even fewer photons exist above $114 \mathrm{eV}$. 
We conclude that the hot gas may be similar to that in the halo of our Galaxy and collisionally ionized (e.g., Sembach \& Savage 1992). It might be heated by gas cloud collisions and supernovae explosions which eject disk gas into the outer regions of the halo (e.g., Fan \& Ikeuchi 1992, Shapiro \& Benjamin 1991). Gas heated to a million degrees cools through radiative and dielectronic recombination on time scales of $t_{c} \sim 10^{4} n_{e}^{-1} y r$, where $n_{e}$ is the electron density (Shapiro \& Moore 1976). The same O VI $2 \mathrm{~s}-2 \mathrm{p}$ transition studied in this paper is responsible for the large cooling peak at $T \sim 10^{5} \mathrm{~K}$ (Cox \& Tucker 1969). To sustain collisionally ionized O VI absorption systems, the densities must be very low $\left(\ll 10^{-2} \mathrm{~cm}^{-3}\right)$, or the heating sources must be very strong.

\subsection{Density of Baryons in Hot Gas}

Hot gas with strong O VI lines could be the largest portion of all baryons, exceeding the $\simeq 7 \%$ in local stars and stellar remnants, and the $\simeq 7 \%$ seen in cool gas as damped Ly $\alpha$ systems at $z \simeq 3$ (Wolfe et al. 1995). The missing baryons might be condensed (e.g. brown dwarfs, black holes), or diffuse hot ionized gas in clouds which are the subject of this paper. Gas which is hotter than $T \geq 10^{6} \mathrm{~K}$ may be seen for the first time since O VI lines are then stronger than those of C IV (Verner, Tytler \& Barthel 1994, hereafter VTB94).

The missing baryons could also be in the IGM, and too hot to show ultraviolet lines. COBE limits on the Compton distortion $y$ parameter limit the temperature and density of a hot and dense IGM, but even if all baryons were in the IGM, the maximum temperature is still a few $\mathrm{keV}$ (few $10^{7} \mathrm{~K}$; Wright et al 1994:Barcons, Fabian \& Rees 1991), which is still too hot to show UV absorption lines.

Many of the missing baryons could be in gas which is cooler. O VI is most prevalent at $T=3 \times 10^{5} \mathrm{~K}$ (VTB94). By $10^{6} \leq T \leq 4 \times 10^{6} \mathrm{~K} \mathrm{O}$ VI lines will be stronger than those from C IV or N V, and for highest temperatures C IV and N V will not be visible at all (VTB94). We will only see the part of the hot gas which is enriched with metals, This could include most hot gas if the heating is associated with supernovae, galaxy formation and mergers, and if all galaxies enrich the gas in their surroundings as occur in clusters.

\subsection{Past Searches}

Although there have been no traditional surveys for individual O VI lines in QSO spectra because of severe blending of lines in the $\operatorname{Ly} \alpha$ forest, the following absorption line studies have observed O VI and provide strong evidence that O VI absorption is both real and common.

Hartquist \& Snijders (1982) showed that O VI is common in absorption systems which are

very close to QSOs, the "associated" C IV systems. Tytler \& Barthel (unpublished) found that most associated systems with strong $\mathrm{C}$ IV lines $\left(W_{r}(C I V) \geq 5 \AA\right)$ have O VI, and O VI is also 
common in BAL systems (Hamann et al. 1995). In all these cases the O VI is strong because the absorbers are near individual QSOs. Therefore the gas is probably photoionized, which shows that many QSOs do emit above $114 \mathrm{eV}$. This radiation does escape into the QSO's galaxy, but not necessarily into the IGM.

Lu \& Savage (1993) formed a single composite spectrum of the absorption lines from typical high redshift $\left(z_{a b s} \simeq 2.8\right)$ absorption systems which show relatively strong $\mathrm{C}$ IV lines. They found $\mathrm{O}$ VI in systems which had C IV, but they could not determine the fraction of C IV systems with O VI. Some had low ionization lines, while others did not. They did not detect N V lines, and deduced $N(\mathrm{O}$ VI $) / N(\mathrm{~N} \mathrm{~V}) \geq 4.4$, which implies $T \geq 2.5 \times 10^{5} \mathrm{~K}$ for collisional ionization.

Bahcall et al. (1993) note that O VI is seen in three of the metal line systems in their HST quasar spectra, one of which was an associated absorber. Bergeron et al. (1994) note that 4 of 5 metal systems show O VI, but they are unable to determine if the gas is collisionally ionized or photoionized. These results all suggest that O VI systems are as common or more common than C IV systems (several per QSO). We will now determine how common.

First we show that we can identify O VI doublets in the Ly $\alpha$ forest. We choose to work with HST UV spectra because the forest is less dense at the lower redshifts of HST spectra compared to the higher redshifts visible from the ground. We measure the frequency of O VI systems, and we try to determine the level of ionization, and whether the gas is photoionized or collisionally ionized. Finally we estimate the contribution that hot gas makes to the cosmological mass density of baryons.

\section{OXYGEN VI SEARCH}

The Hubble Space Telescope Archive provides a resource to search for O VI absorption. In particular, the medium resolution $(\mathrm{R} \approx 1300)$ gratings of the Faint Object Spectrograph have both the sensitivity and the resolving power to identify weak metal lines in absorption. Spectra are chosen by the QSO properties (magnitude and redshift), gratings, and exposure time. Since the search for O VI is done exclusively in the Lyman- $\alpha$ forest, high signal-to-noise is necessary.

To ensure the reality of O VI absorption, strict rules are applied to line lists. These rules incorporate measured wavelength, equivalent width, and $1 \sigma$ errors. Once the ground rules are determined, a computer algorithm is responsible for identifying systems which exhibit O VI absorption. We use a computer to automatically find O VI and to maintain objectivity and repeatability. Physical significance is measured by applying the same rules to false sets of rest wavelengths and comparing results with the true set. 


\subsection{Processing the HST Spectra}

We consider spectra from only the FOS high resolution gratings G130H, G190H and G270H. The G160L does not have enough resolution, and there were no suitable GHRS G140L spectra. Table 1 shows the 11 QSOs chosen for the O VI search. Most of the spectra are from the G190H or $\mathrm{G} 270 \mathrm{H}$ gratings, because few $\mathrm{G} 130 \mathrm{H}$ spectra have adequate $\mathrm{S} / \mathrm{N}$. There are 8 steps prior to the O VI search,

1. We use the following rules to ensure that the $z_{e m}$ of each QSO is sufficient to place O VI well onto the FOS gratings:

$$
z_{e m} \geq \begin{cases}0.3 & \text { for } \mathrm{G} 130 \mathrm{H} \\ 0.65 & \text { for } \mathrm{G} 190 \mathrm{H} \\ 1.25 & \text { for } \mathrm{G} 190 \mathrm{H}\end{cases}
$$

2. We obtained each spectrum and its error array from the HST Archive.

3. Coadd spectra which have multiple exposures.

4. Find all the absorption lines from the interstellar medium of our Galaxy. We shift each spectrum so that these lines give a mean velocity of zero (Schneider et al. 1993). We list the offsets added to each spectrum in Table 1.

5. Fit a continuum to each spectrum manually. Due to the subjectivity of continuum fitting, the systematic errors introduced in the measured equivalent widths are difficult to calculate. However, we assume that other errors in our final results greatly outweigh the errors in the continuum fits.

6. Convert the spectrum and its error array into a spectrum which gives the minimum equivalent width of unresolved lines of specified significance level.

7. Find all $5 \sigma$ lines in a spectrum. There are typically $40-80$. Measure their equivalent widths $W, \sigma(W)$, and wavelengths.

8. Search the literature for all known absorption systems. Identify lines which belong to these systems, and remove them from the sample in which we will search for O VI.

\subsection{Search for O VI Lines}

There are no published systematic searches for individual spectral lines in the Ly $\alpha$ forest region because the large number of forest lines and the high incidence of line blending lead to numerous false identification. We require a search which has the desirable attributes of a medical screen for a rare disease: (1) high sensitivity to minimize the number of missed O VI systems (false negatives), and (2) high specificity to minimize the number of false positive identifications. We 
use a computer code to increase the sensitivity, accuracy, completeness and speed of the survey, and use rules to limit the number of false positives to about $20 \%$ of the sample. We would prefer to have fewer false positives, but this is only possible if we consider just the very strongest lines. Then we miss most O VI lines, and we cannot estimate their overall frequency.

False identifications can be noise features, or real lines with other identifications, especially Ly $\alpha$. We use three criteria to limit the number of false identification: line significance, redshift agreement, and equivalent width ratios. In all detections, both O VI lines must be detected above the $2 \sigma$ equivalent width limit. Furthermore, their measured equivalent width ratio must lie within $1 \sigma$ of the theoretical boundaries.

The procedures we use to find O VI lines in certain and possible redshift systems are described below.

\subsubsection{Search for O VI Lines in Certain Redshift Systems}

In published, or new metal line systems (e.g. with Mg II, C IV or Lyman limits) which are considered certain, we look for $2 \sigma \mathrm{O}$ VI lines. There are about $0-3$ such systems per QSO, so the chance of false positive identification of both O VI lines is $\leq 3 \%$ per QSO. We measure lines associated with the absorption systems and calculate the mean redshift, which can vary from the published absorption redshift because of wavelength offsets. We identify all additional lines, including O VI, which match the criteria of the next section.

\subsubsection{Search for O VI Lines in Uncertain Redshift Systems}

We use the following procedure to find interesting lines, identify possible new redshift systems and then reject unreasonable ones.

1. We search for all systems with at least two $5 \sigma$ lines from $\operatorname{Ly} \alpha, \operatorname{Ly} \beta$, Ly $\gamma$ or O VI. Lines are accepted into a system if their redshift differs from the weighted mean $z_{\text {ave }}$ by less than $0.0005\left(\sim 80 \mathrm{~km} \mathrm{~s}^{-1}\right)$, which is sufficient to include $93 \%$ of lines in metal systems identified by Bahcall et al. (1993). The weighted mean $z_{\text {ave }}$ is defined as

$$
z_{\text {ave }} \equiv \frac{\sum_{i} w_{i}^{2} z_{i}}{\sum_{i} w_{i}^{2}}
$$

where $w_{i}$ is the weight placed on the redshift of each line:

$$
w_{i}=\left\{\begin{array}{ll}
\frac{W_{o b s}}{\sigma(W)} & W_{o b s}<0.79 \mathrm{FWHM} \\
\frac{0.79 F W H M}{\sigma(W)} & W_{o b s} \geq 0.79 \mathrm{FWHM}
\end{array},\right.
$$


where FWHM is the spectral resolution of each grating in $\AA$. We define the equivalent width cut-off as the limit for unsaturated lines. Once a line becomes saturated, the accuracy of the line center depends only on the $\mathrm{S} / \mathrm{N}$ of the spectrum. Lines which exceed the saturation criterion are marked in Table 4.

2. We calculate expected positions of the remaining lines in these system, and we measure wavelengths and equivalent widths, or limits for these lines.

3. We search for additional identifications for the new $2 \sigma$ lines amongst all systems with at least two $5 \sigma$ lines.

4. As a further constraint, we use the ratios

$$
R_{H I}=\frac{W(1215)}{W(1025)}
$$

and

$$
R_{O V I}=\frac{W(1032)}{W(1038)}
$$

If $\operatorname{Ly} \beta$ (1025) or $\mathrm{O} \mathrm{VI}(1038)$ is not present, then the ratios are calculated using the $2 \sigma$ equivalent width limits. A ratio is judged acceptable if it falls between $1-\sigma(R)$ and $2+\sigma(R)$ for $\mathrm{O} \mathrm{VI}$, and $1-\sigma(R)$ and $5.27+\sigma(R)$ for $\mathrm{H} \mathrm{I}$, where $\sigma(R)$ is the error on the ratio obtained from the errors on the individual $W$ values, and the numerical values are for unsaturated and saturated lines. We discard systems which do not have at least one acceptable ratio.

We wrote a computer code to follow the above procedures, and we ran this code with both true and false rest wavelengths. The false wavelengths did not have the same ratios as the true wavelengths of any strong lines. We then counted the number of O VI systems identified with both true and false wavelengths. The above procedures were then adjusted to give a true/false ratio of 5:1. We also ran tests with true rest wavelengths and random absorption line positions, which gave the same result.

Unfortunately, we did not accept lone O VI lines, because the number of false identifications was similar to the true number. In figure 1, we show the number Our simulations show that we must insist that all systems have $\geq 3$ lines to keep the expected number of false O VI identifications $<1$ per spectrum. Simulations indicate that Ly $\alpha$ should be stronger than O VI in either collisional or photo- ionization (VTB94), so we use lines of the Lyman series to confirm O VI identifications. Although we exclude possible O VI detections, it is necessary to detect a third line to keep false identifications to a minimum. A Lyman line must be detected at the O VI redshift with the following stipulations:

1. If Ly $\alpha$ exists and $R(H)$ is acceptable, then the system is accepted. 
2. If $\operatorname{Ly} \beta$ exists without a $\operatorname{Ly} \alpha$, the system is discarded.

3. If $\operatorname{Ly} \beta$ exists and has an acceptable equivalent width ratio with $\operatorname{Ly} \gamma$, and $\operatorname{Ly} \alpha$ cannot be checked, the system is accepted.

No method will throw out all false systems and keep all real ones. For example, consider pairs of $5 \sigma$ lines which are identified as $\operatorname{Ly} \alpha$ and $\operatorname{Ly} \beta$. Simulations show that such pairs do occur commonly, even in HST spectra at low $z$ : we expect 2 false pairs of $5 \sigma$ lines in every QSO. But in real data we see about 6 such $\operatorname{Ly} \alpha-\operatorname{Ly} \beta$ pairs per QSO, so $67 \%$ are real. We look for $2 \sigma$ O VI lines in each such system.

\section{FREQUENCY OF OXYGEN VI}

In Table 2, we list the number of O VI systems identified with three different sample criteria. In Sample A, the absorption system identified with O VI must have at least three lines with a redshift within $\Delta z=0.0005$ of the weighted mean redshift of the system. All lines must have a significance level $\geq 2 \sigma$. Sample B is a subset of A where at least two of the lines must have a significance level of $\geq 5 \sigma$. Sample $\mathrm{C}$ is a subset of $\mathrm{A}$, with the constraint that both lines of the O VI doublet must have a rest equivalent width $W_{\text {rest }} \geq 0.21 \AA$, which is the minimum $2 \sigma$ rest equivalent width which could be seen in all the spectra. Sample $\mathrm{C}$ is the most stringent and complete sub-sample and will be used to measure the density of O VI systems.

Also shown in the same table is the number of O VI identifications per object using false rest wavelengths. Simulations were run with 10 sets of false wavelengths for $\operatorname{Ly} \alpha, \operatorname{Ly} \beta, \operatorname{Ly} \beta$ and O VI. We used the same criteria as with the real wavelengths. The average number of false identifications is shown in the table, and gives an estimate of the number of identifications in the true sample which are due to chance.

Samples A and B differ by less than we expected, because there is only one system in A that does not satisfy the requirements of B. But A contains twice as many false IDs as B, so B is a significantly cleaner sample. The line density is actually higher in B than A, but the difference is not significant.

With 11 objects in this preliminary survey, we can estimate the number of O VI absorbers per unit redshift.

$$
N(z)=\frac{\text { Total True }- \text { Total False }}{\Delta z_{t o t}}
$$

where

$$
\sigma_{N}(z)=\frac{\sqrt{\text { Total True }+ \text { Total False }}}{\Delta z_{\text {tot }}}
$$

where $\Delta z_{\text {tot }}=4.73$. 
Table 3 contains system number densities for a variety of absorption systems. The first three are our samples of O VI, and the fourth is a sample of $\operatorname{Ly} \alpha-\mathrm{Ly} \beta$ pairs in which we found W(1025) $\geq 0.30 \AA$ For comparison with other samples, we use Sample C, which is most complete. At low $z$ systems with O VI are as common as the other main types of system: Mg II, C IV and LLS.

\subsection{Individual Systems with O VI}

Table 4 shows the twelve systems of Sample A. For each of these systems we searched for all other strong lines which would appear in the observed wavelength range, including: C IV 1548, 1550, N V 1238, 1242, Si III 1206, Si II 1190, 1193, 1260, Si IV 1393, 1402, N III 989, C III 977, and C II 1334. Two of these systems show H I and O VI only, but all the others show at least one other element, and have $\geq 5$ lines each. Lines are accepted into a system if their redshift is within \pm 0.0005 of the weighted mean.

In Figure 2 we show the absorption line positions on the HST spectra. Note that the Ly $\beta$, O VI(1032) and O VI(1038) lines form a well resolved uniformly spaced triplet, which helps visual identifications.

For each of the 12 O VI systems of Sample A, Figure 3 shows enlarged plots of Ly $\alpha$, Ly $\beta$, O VI and C IV all on a velocity scale given by the listed mean redshift. We do not show lines which are below $2 \sigma$ significance. Line widths are all set by the FOS resolution. Comments on individual systems are in the appendix.

\subsection{Contamination of O VI(1038)}

Lu \& Savage (1993) point out that O VI 1037.62 can be contaminated by C II 1036.34 and O I 1039.23, especially in low ionization systems.

Lines O VI(1038) and C II(1036) are separated by $\Delta \lambda=1.32$ so to resolve these lines we need spectra with resolution $R \geq 785$. The FOS high resolution spectra have $\mathrm{R} \approx 1300$ so we would

expect, assuming usual $b$ parameters $\leq 75 \mathrm{~km} \mathrm{~s}^{-1}$, that these lines should be resolved in this survey. We do just resolve these lines, and we fitted each to get their individual equivalent widths which are listed in Table 5.

As a check we measured lines C II 1334.53 and O I 1302.17 which should both be stronger than the contaminants C II 1036.34 and O I 1039.23 because their $\lambda f$ values are larger by 1.3 and 6.6 times respectively. We follow this procedure:

1. Measure width of $\mathrm{C} \operatorname{II}(1334)$.

2. Calculate line width of C II(1036) with ratio of oscillator strengths, assuming lines are linear. 
3. Measure total width from 1036-1038 A.

4. Subtract calculated C II(1036) from the combined width of step 3

In Table 5, we list the equivalent widths of O VI(1032), O VI (1036-1038) and C II(1334). The O VI 1036-1038 widths in Table 5 includes both O VI and C II, whereas those in Table 4 are for O VI alone. We detect C II(1334) in four O VI systems, and for each of these systems, we subtract W(C II,1036) from $W_{r}(1036-1038)$ to calculate the "subtracted" doublet ratio. If absorption is due to O VI and C II alone, this would provide the correct width of O VI(1038). For all the systems of Sample A, both the fitted and subtracted doublet ratios fall within the allowed $1 \sigma$ errors. The fitting procedure contains less steps and is subject to less uncertainty than the subtracting precedure. Since the spectra of this survey have adequate resolution to separate C II(1036) and O VI(1038), we conclude that the measured widths and ratios obtained by fitting O VI and C II (Table 4) independently are more accurate.

The other possible contamination due to O I is ruled out. O I $\lambda 1302$ was not detected in any systems associated with O VI. So the contribution from the weaker O I(1039) is safely assumed negligible.

\subsection{Column Densities and Velocity Dispersions}

We employ the doublet ratio method to estimate column densities and velocity dispersions where possible. Table 6 shows our calculations for O VI, C IV, and H I in systems where two or more ions are present. We calculate the $1 \sigma$ errors in Table 6 by allowing the equivalent widths to assume any value with their $1 \sigma$ errors and taking the extreme results as limits. This is an over estimation of the random errors which partially compensates for the systematic errors of measuring equivalent widths of unresolved lines in the Ly $\alpha$ forest. Wavelengths and f-values used in the column density calculations were taken from Morton (1991).

As seen from Table 6, our search was only sensitive to column densities $\log (\mathrm{N})>14.4$. This is consistent with the minimum equivalent width threshold, $W_{r}=0.21 \AA$. Figure 4 shows the allowable column densities of a single component for a given doublet ratio. Our survey of O VI is not sensitive to linear single component absorbers. High velocity dispersion $\left(>100 \mathrm{~km} \mathrm{~s}^{-1}\right)$ is strong evidence for multiple absorbers in the system.

\subsection{Collisional or Photo- ionization?}

HST FOS spectra do not have enough spectral resolution to give accurate column densities and velocity dispersions, so it is difficult to determine if the gas is photoionized or collisionally ionized. So we use the relative strengths of the O VI, C IV, and Ly $\beta$ lines to indicate the level of 
ionization. We define two equivalent width ratios:

$$
R_{O B}=\frac{O V I(1032)}{\operatorname{Ly} \beta}
$$

and

$$
R_{O C}=\frac{O V I(1032)}{C I V(1548)}
$$

In Table 7 we give values for these ratios. $\operatorname{Ly} \beta$ is seen in 11 systems, and C IV in 8.

Seven out of the eight systems in which C IV and O VI are detected have similar ratios: $R_{O C}<1.2$ and $R_{O B}<1$ corresponding to medium ionization. Although C IV and O VI are both detected, C IV is the dominant ion and the system should be labeled as such. Five of the seven medium ionization systems exhibit multiple low ionization lines (i.e. C II, C III, N III, Si II, $\mathrm{Si}$ III). The ionization energies in each of the five systems range from $11 \mathrm{ev}$ to $114 \mathrm{eV}$. Such a wide range of ionizations, and therefore a large variety of ions, cannot be modeled with pure collisional ionization in a single simple cloud. As was restated by Verner and Yakolev (1990), the large variety of ions found in absorption systems is strong evidence that power law ionizing radiation is present. The eighth system, at $z_{a b s}=1.08$ of PG $1206+4557$, has $R_{O C}=4.17$ and $R_{O B}=2.02$. This system has significantly different values and is classified as high ionization. Only collisional ionization at high temperatures, $T>10^{5} \mathrm{~K}$, or extreme photoionization, $\chi>0.1$, can produce the measured ratios ( $\chi$ is the ratio of the number of photons with energies above the Lyman edge to the number of particles). Since C IV is so weak and N V is undetected, we believe that the system is collisionally ionized.

In the remaining four systems in which O VI was detected, none had a corresponding $\mathrm{C} \mathrm{IV}$ detection. Each was assigned an upper limit $2 \sigma$ equivalent width associated with the non-detection of C IV. These systems are marked on Figure 5 with arrows starting at the lower limit of the ratio. In the system $z_{a b s}=0.746$ of PKS 1424-1120, neither C IV nor Ly $\beta$ are detected and a lower limit is assigned for each ratio. These systems are labeled as high ionization since O VI is the dominant metallic ion.

\subsection{Cosmological Mass Density}

The cosmological mass density $\Omega_{O V I}(z)$ of $\mathrm{O}$ VI is defined as the comoving mass density of $\mathrm{O}$ VI in terms of the current critical density.

$$
\Omega_{O V I}=\frac{m_{O V I}}{\rho_{c} c H_{O}^{-1}} \frac{\sum_{i} N_{i}(O V I)}{\sum_{i} \Delta X_{i}}
$$

where $m_{O V I}$ is mass of the Oxygen ion, $\rho_{c}$ is the current critical density, $H_{o}$ is the current Hubble parameter, $N_{i}(O V I)$ is total O VI column density towards the $i$ th QSO over an absorption path distance $\Delta X_{i}$ defined as 


$$
\Delta X= \begin{cases}\frac{1}{2}\left\{\left[\left(1+z_{\max }\right)^{2}-1\right]-\left[\left(1+z_{\min }\right)^{2}-1\right]\right\} & \left(q_{0}=0\right) \\ \frac{2}{3}\left\{\left[\left(1+z_{\max }\right)^{3 / 2}-1\right]-\left[\left(1+z_{\min }\right)^{3 / 2}-1\right]\right\} & \left(q_{0}=0.5\right)\end{cases}
$$

Using values of the redshift range in Table 2,

$$
\sum_{i} \Delta X_{i}= \begin{cases}9.24 & \left(q_{0}=0\right) \\ 6.60 & \left(q_{0}=0.5\right)\end{cases}
$$

The standard error of the mass density is estimated as

$$
\sigma_{\Omega}=\frac{1}{\left(1-\frac{1}{m}\right)^{1 / 2}} \frac{\sqrt{\left.\sum_{i}\left[N_{i}(O V I)-\langle N(O V I)\rangle\right]^{2}\right)}}{\Delta X_{t o t}},
$$

where $\mathrm{m}$ is the total number of absorption systems in the sample, and $\langle N(O V I)\rangle$ is the mean column density of the sample.

Lower limits of the column density in each absorption system are calculated with the assumption that the O VI absorption features are unsaturated. The column density of the absorber is then proportional to the rest equivalent width (Spitzer 1978)

$$
N\left(\mathrm{~cm}^{-2}\right)=\frac{1.13 \times 10^{20} W(\AA)}{\lambda^{2}(\AA) F_{i j}},
$$

where $F_{i j}$ is the oscillator strength of the transition $\lambda$ is the rest wavelength of the transition $\mathrm{W}$ is the rest equivalent width. The absorption features of O VI $\lambda 1038$ are weaker and less saturated. Using the six systems of Sample C and the rest equivalent width of O VI(1038), we obtain

$$
\sum_{i} N_{i}(O V I) \geq 5.9 \times 10^{15} \mathrm{~cm}^{-2}
$$

We arrive at an estimate for the cosmological mass density of O VI at $z_{\text {ave }}=0.9$,

$$
\Omega_{O V I} \geq \begin{cases}1.4 \pm 0.4 \times 10^{-8} h^{-1} & \left(q_{0}=0\right) \\ 2.0 \pm 0.6 \times 10^{-8} h^{-1} & \left(q_{0}=0.5\right)\end{cases}
$$

where $H_{o}=100 h \mathrm{~km} \mathrm{~s}^{-1} \mathrm{Mpc}^{-1}$.

\subsection{Limit on Cosmic Metallicity}

It is now straightforward to place a lower limit on the mean cosmological metallicity at $z_{\text {ave }}=0.9$. If the cosmic metallicity is too low, all the baryons in the universe are needed to 
account for the O VI seen in this survey. Let $\zeta(\mathrm{z})$ be defined as the ratio of mean metallicity at redshift $z$ to the solar metallicity,

$$
\zeta(z)=\mu\left(\frac{H}{O}\right)_{\text {Solar }}\left(\frac{O}{O V I}\right) \frac{\Omega_{O V I}(z)}{\Omega_{b}}
$$

where $\mu$ is the mean molecular weight, which equals 1.3 for a mixture of $25 \%$ Helium and $75 \%$ Hydrogen by mass; $(H / O)_{\text {Solar }}=1174.9$ (Anders \& Grevesse 1989); and $\Omega_{b}$ is the cosmological baryon mass density. The ionization fraction $\mathrm{O} / \mathrm{O} \mathrm{VI}$ is impossible to calculate for each absorption system with the current data, but a lower limit is placed on the metallicity by assuming O/O VI $=1$. A recent measurement of deuterium at high redshift by (Tytler \& Fan 1994) yields $\Omega_{b}^{-1}(z)=0.023 h^{-2}$.

$$
\zeta(z) \geq \begin{cases}8.9 \pm 2.7 \times 10^{-4} h & \left(q_{0}=0\right) \\ 1.4 \pm 0.4 \times 10^{-3} h & \left(q_{0}=0.5\right) .\end{cases}
$$

The calculation is subject to small number statistics, and we can estimate the sensitivity of the lower limit by removing one system of Sample C. If we discard the system with the largest equivalent width $\left(z_{a b s}=0.927\right.$ towards $\left.1206+4557\right)$, the lower limit is reduced by $35 \%$. The calculated lower limit is a safe estimate for the following reasons: We have assumed that all Oxygen at $z_{\text {ave }}=0.9$ is in the form of O VI, that all absorption is linear, and that we have detected all $\mathrm{O}$ VI along the absorption path.

\section{SUMMARY}

O VI absorption is as common at $z_{\text {ave }}=0.9$ as $\mathrm{C}$ IV and $\mathrm{Mg}$ II absorption. Using a minimum rest equivalent width of $W_{r}=0.21 \AA$ and accounting for the average number of chance coincidences per spectra, the number density of O VI absorbers per redshift is

$$
\left\langle N_{O V I}(z=0.9)\right\rangle=1.0 \pm 0.6
$$

Using a systematic computer search, we found 12 redshifts with $\geq 2 \sigma$ equivalent widths at O VI wavelengths. The O VI systems were categorized as either medium or high ionization, Seven systems are medium ionization, with 5 showing multiple low ionization lines. The 5 high ionization systems have O VI stronger than both $\mathrm{C}$ IV and Ly $\beta$.

We summed over all O VI absorption with widths above the threshold $W_{r}>0.21 \AA$ to estimate the cosmological mass density at $z=0.9$. Since we could not resolve velocity structure, we calculated a lower limit by assuming linear absorption,

$$
\Omega_{O V I} \geq \begin{cases}1.4 \pm 0.4 \times 10^{-8} h^{-1} & \left(q_{0}=0\right), \\ 2.0 \pm 0.6 \times 10^{-8} h^{-1} & \left(q_{0}=0.5\right)\end{cases}
$$


We proceed further by assuming all Oxygen is in the form of O VI and place a lower limit on the metallicity in solar units at $z=0.9$,

$$
\zeta(z) \geq \begin{cases}8.9 \pm 2.7 \times 10^{-4} h & \left(q_{0}=0\right) \\ 1.4 \pm 0.4 \times 10^{-3} h & \left(q_{0}=0.5\right)\end{cases}
$$

The survey for hot gas, such as O VI, must be pursued in the future. In addition to better statistics at low redshift, the survey should be extended to high redshift to measure changes in the abundance of hot gas. Ions with higher energies, such as Ne VIII and Mg X, should be studied. The launch of the Hubble Space Telescope has allowed us to quantify the amount of $100 \mathrm{eV}$ intergalactic gas for the first time.

We are grateful to Chris Davis for assisting in the reduction of the archived HST spectra. 


\begin{tabular}{|c|c|c|c|c|c|c|}
\hline $\mathrm{QSO}(1950)$ & Name & $z_{e m}$ & $\mathrm{~V}$ & FOS Grating & Offset $(\AA)$ & Exposure (s) \\
\hline \multirow[t]{2}{*}{$0122-0021$} & PKS 0122-00 & 1.070 & 16.70 & $190 \mathrm{H}$ & +1.3 & 3000 \\
\hline & & & & $270 \mathrm{H}$ & +0.8 & 720 \\
\hline \multirow{3}{*}{ 0454-2203 } & PKS 0454-22 & 0.534 & 16.1 & $130 \mathrm{H}$ & 0.0 & 6500 \\
\hline & & & & $190 \mathrm{H}$ & 0.0 & 5900 \\
\hline & & & & $270 \mathrm{H}$ & 0.0 & 2000 \\
\hline \multirow[t]{2}{*}{$1206+4557$} & PG $1206+459$ & 1.158 & 15.79 & $190 \mathrm{H}$ & +0.8 & 4500 \\
\hline & & & & $270 \mathrm{H}$ & +0.9 & 1000 \\
\hline \multirow[t]{2}{*}{$1317+2743$} & Ton 153 & 1.022 & 15.98 & $190 \mathrm{H}$ & +1.5 & 4500 \\
\hline & & & & $270 \mathrm{H}$ & +1.4 & 1000 \\
\hline \multirow[t]{2}{*}{$1352+0106$} & PG $1352+011$ & 1.121 & 16.03 & $190 \mathrm{H}$ & +2.3 & 5300 \\
\hline & & & & $270 \mathrm{H}$ & +1.6 & 1150 \\
\hline \multirow[t]{2}{*}{$1407+2632$} & PG $1407+265$ & 0.944 & 15.73 & $190 \mathrm{H}$ & +1.5 & 4250 \\
\hline & & & & $270 \mathrm{H}$ & +1.9 & 1125 \\
\hline \multirow[t]{2}{*}{$1424-1150$} & PKS 1424-11 & 0.806 & 16.49 & $190 \mathrm{H}$ & +1.5 & 6600 \\
\hline & & & & $270 \mathrm{H}$ & +1.7 & 1900 \\
\hline $1435+6349$ & $\mathrm{~S} 4-1435+63$ & 2.068 & 15.0 & $270 \mathrm{H}$ & 0.0 & 3200 \\
\hline \multirow[t]{2}{*}{$1522+1009$} & PG $1522+101$ & 1.321 & 15.74 & $190 \mathrm{H}$ & +0.6 & 9500 \\
\hline & & & & $270 \mathrm{H}$ & +1.9 & 2650 \\
\hline \multirow[t]{2}{*}{$1634+7037$} & PG $1634+706$ & 1.334 & 14.90 & $190 \mathrm{H}$ & +0.9 & 5300 \\
\hline & & & & $270 \mathrm{H}$ & +0.7 & 6750 \\
\hline $2340-0340$ & PKS 2340-03 & 0.896 & 16.02 & $190 \mathrm{H}$ & +0.2 & 4100 \\
\hline
\end{tabular}

Table 1: List of Quasar Spectra 


\begin{tabular}{|c|c|c|c|c|c|c|c|c|c|c|}
\hline \multirow[t]{3}{*}{ Object } & \multicolumn{2}{|c|}{ Lyman- $\alpha, \beta$} & \multicolumn{6}{|c|}{ O VI(1032,1038) } & \multirow{3}{*}{$\begin{array}{c}\text { Redshift } \\
\text { Range } \\
(\Delta z)\end{array}$} & \multirow{3}{*}{$\begin{array}{l}\text { Minimum } \\
\text { Observed } \\
\text { Width }(\AA)\end{array}$} \\
\hline & \multirow[t]{2}{*}{ True ID } & \multirow[t]{2}{*}{ False ID } & \multicolumn{3}{|c|}{ True ID } & \multicolumn{3}{|c|}{ False ID } & & \\
\hline & & & $\mathrm{A}$ & $\mathrm{B}$ & $\mathrm{C}$ & $\mathrm{A}$ & $\mathrm{B}$ & $\mathrm{C}$ & & \\
\hline 0122-0021 & 5 & 2.2 & 2 & 2 & 2 & 0.2 & 0.1 & 0.0 & $0.61-1.07$ & 0.31 \\
\hline $0454-2203$ & 4 & 0.7 & 1 & 1 & 1 & 0.4 & 0.1 & 0.1 & $0.31-0.53$ & 0.28 \\
\hline $1206+4557$ & 6 & 1.1 & 3 & 2 & 2 & 0.2 & 0.2 & 0.1 & $0.61-1.16$ & 0.24 \\
\hline $1317+2743$ & 2 & 0.6 & 0 & 0 & 0 & 0.3 & 0.2 & 0.1 & $0.61-1.02$ & 0.21 \\
\hline $1352+0106$ & 5 & 1.1 & 1 & 1 & 1 & 0.3 & 0.3 & 0.2 & $0.61-1.12$ & 0.23 \\
\hline $1407+2632$ & 6 & 0.3 & 0 & 0 & 0 & 0.1 & 0.1 & 0.1 & $0.61-0.94$ & 0.31 \\
\hline $1424-1150$ & 2 & 0.1 & 2 & 2 & 0 & 0.0 & 0.0 & 0.0 & $0.61-0.81$ & 0.29 \\
\hline $1435+6349$ & 0 & 0.6 & 0 & 0 & 0 & 0.7 & 0.2 & 0.2 & $1.61-2.08$ & 0.23 \\
\hline $1522+1009$ & 2 & 0.6 & 0 & 0 & 0 & 0.7 & 0.4 & 0.3 & $0.61-1.32$ & 0.16 \\
\hline $1634+7037$ & 1 & 0.2 & 2 & 2 & 0 & 0.4 & 0.1 & 0.1 & $0.75-1.33$ & 0.17 \\
\hline $2340-0340$ & 3 & 0.4 & 1 & 1 & 0 & 0.2 & 0.1 & 0.1 & $0.61-0.90$ & 0.25 \\
\hline Total & 39 & 7.9 & 12 & 11 & 6 & 3.5 & 1.8 & 1.3 & 4.73 & \\
\hline
\end{tabular}

Table 2: Frequency Of Absorption Systems with O VI Doublets

\footnotetext{
A: System must have at least three lines with a redshift within $\Delta z=0.0005$ of the mean redshift of the system. All lines must be $\geq 2 \sigma$.

$\mathrm{B}$ : Subset of $\mathrm{A}$ where at least two lines are $\geq 5 \sigma$.

C: Subset of B where both O VI lines have a rest equivalent width $\geq 0.21 \AA$.
} 


\begin{tabular}{|c|c|c|c|c|c|c|}
\hline Ion & Sample & True & False & $\bar{z}$ & $\mathrm{~N}(\mathrm{z})$ & ref \\
\hline & & Low & Redshift & & & \\
\hline \multirow[t]{3}{*}{$\mathrm{O} \mathrm{VI}$} & $\mathrm{A}$ & 12 & 3.5 & 0.9 & $1.8 \pm 0.8$ & 1 \\
\hline & B & 11 & 1.8 & 0.9 & $1.9 \pm 0.7$ & 1 \\
\hline & $\mathrm{C}$ & 6 & 1.3 & 0.9 & $1.0 \pm 0.6$ & 1 \\
\hline $\mathrm{HI}$ & $\operatorname{Ly} \alpha-\operatorname{Ly} \beta$ & 39 & 8 & 0.9 & $6.5 \pm 1.4$ & 1 \\
\hline $\mathrm{HI}$ & $\operatorname{Ly} \alpha$ & $\mathrm{W}(1215)$ & )$>0.32 \AA$ & 0.7 & $21.8 \pm 2.1$ & 2 \\
\hline $\mathrm{HI}$ & LLS & $\tau>$ & $>0.4$ & 0.9 & $1.1 \pm 0.3$ & 2 \\
\hline C IV & $\mathrm{W}(15$ & 48) $>0.3$ & $30 \AA$ & 0.3 & $0.87 \pm 0.43$ & 2 \\
\hline $\mathrm{Mg}$ II & $\mathrm{W}(27$ & $96)>0.6$ & $60 \AA$ & 0.5 & $0.26 \pm 0.08$ & 3 \\
\hline Mg II & $\mathrm{W}(27$ & $96)>0.3$ & $30 \AA$ & 0.9 & $1.0 \pm 0.25$ & 4 \\
\hline
\end{tabular}

Table 3: Number Density Of Various Absorption Systems

References. - (1) This Paper; (2) Bahcall et al. 1993; (3) Tytler et al. 1987; (4) Fan 1995. 
Table 4. Oxygen VI System Line List ${ }^{\mathrm{a}}$

\begin{tabular}{|c|c|c|c|c|c|c|}
\hline $\begin{array}{l}\text { Wavelength } \\
(\AA)\end{array}$ & ID & $z_{o b s}$ & $\begin{array}{c}W_{o b s} \\
(\AA)\end{array}$ & $\begin{array}{c}\sigma(W) \\
(\AA)\end{array}$ & $\mathrm{SL}^{\mathrm{b}}$ & $\mathrm{LR}^{\mathrm{c}}$ \\
\hline & \multicolumn{2}{|c|}{ 0122-0021 } & \multicolumn{2}{|c|}{$z_{e m}=1.070$} & \multicolumn{2}{|l|}{$\mathrm{V}=16.1^{\mathrm{d}}$} \\
\hline 1 & \multicolumn{5}{|c|}{$z_{\text {ave }}=0.95336, \sigma_{z}=0.00031 \mathrm{~A}, \mathrm{~B}, \mathrm{C}^{\mathrm{e}}$} & \\
\hline 2374.84 & $\operatorname{Ly} \alpha$ & 0.95352 & 2.368 & $0.205^{\mathrm{f}}$ & 11.55 & 1.73 \\
\hline 2003.88 & $\operatorname{Ly} \beta$ & 0.95363 & 1.367 & $0.102^{\mathrm{f}}$ & 13.38 & 2.19 \\
\hline 1900.05 & $\operatorname{Ly} \gamma$ & 0.95370 & 0.623 & 0.122 & 5.11 & \\
\hline 2015.31 & OVI(1032) & 0.95295 & $1.211^{\mathrm{g}}$ & $0.098^{\mathrm{f}}$ & 12.31 & 1.41 \\
\hline 2026.92 & OVI(1038) & 0.95343 & 0.861 & 0.095 & 9.03 & \\
\hline 1908.48 & CIII $(977)$ & 0.95337 & 1.395 & $0.118^{\mathrm{f}}$ & 11.82 & \\
\hline 1933.90 & $\operatorname{NIII}(989)$ & 0.95383 & 0.694 & 0.102 & 6.80 & \\
\hline \multirow[t]{3}{*}{2356.67} & $\operatorname{SiIII}(1206)$ & 0.95331 & 0.467 & 0.200 & 2.35 & \\
\hline & $\mathrm{NV}(1238)$ & & $\leq 0.410^{\mathrm{h}}$ & & & \\
\hline & $\mathrm{NV}(1242)$ & & $\leq 0.410^{\mathrm{h}}$ & & & \\
\hline 2606.32 & CII $(1334)$ & 0.95298 & 0.360 & 0.154 & 2.33 & \\
\hline 3024.09 & CIV (1548) & 0.95329 & 1.989 & $0.180^{\mathrm{f}}$ & 11.05 & 1.13 \\
\hline 3029.12 & CIV(1550) & 0.95330 & 1.763 & $0.180^{\mathrm{f}}$ & 9.79 & \\
\hline \multicolumn{7}{|c|}{$z_{\text {ave }}=0.96686, \sigma_{z}=0.00031 \mathrm{~A}, \mathrm{~B}, \mathrm{C}^{\mathrm{e}}$} \\
\hline 2391.13 & $\operatorname{Ly} \alpha$ & 0.96693 & 1.873 & $0.202^{\mathrm{f}}$ & 9.29 & 2.33 \\
\hline 2017.20 & $\operatorname{Ly} \beta$ & 0.96662 & 0.804 & 0.098 & 8.22 & $0.58^{\mathrm{i}}$ \\
\hline 1912.64 & $\mathrm{Ly} \gamma$ & 0.96664 & 1.394 & $0.116^{\mathrm{f}}$ & 12.04 & \\
\hline 2030.11 & OVI(1032) & 0.96730 & 0.708 & 0.095 & 7.47 & 1.45 \\
\hline \multirow[t]{3}{*}{2041.14} & OVI(1038) & 0.96714 & 0.488 & 0.093 & 5.25 & \\
\hline & $\mathrm{NV}(1238)$ & & $\leq 0.410^{\mathrm{h}}$ & & & \\
\hline & $\mathrm{NV}(1242)$ & & $\leq 0.410^{\mathrm{h}}$ & & & \\
\hline \multirow[t]{3}{*}{3044.48} & CIV(1548) & 0.96646 & 0.629 & 0.180 & 3.49 & $\geq 1.74$ \\
\hline & CIV (1550) & & $\leq 0.36^{\mathrm{h}}$ & & & \\
\hline & \multicolumn{2}{|c|}{ 0454-2203 } & \multicolumn{2}{|c|}{$z_{e m}=0.534$} & $\mathrm{~V}=16.1^{\mathrm{d}}$ & \\
\hline 3 & \multicolumn{5}{|c|}{$z_{\text {ave }}=0.41261, \sigma_{z}=0.00025 \mathrm{~A}, \mathrm{~B}, \mathrm{C}^{\mathrm{e}}$} & \\
\hline 1717.28 & $\operatorname{Ly} \alpha$ & 0.41262 & 0.489 & 0.036 & 13.58 & $0.72^{\mathrm{i}}$ \\
\hline 1448.75 & $\operatorname{Ly} \beta$ & 0.41242 & 0.683 & 0.060 & 11.46 & $\geq 5.85^{\mathrm{i}}$ \\
\hline 1457.61 & OVI(1032) & 0.41251 & 0.851 & $0.059^{\mathrm{f}}$ & 14.38 & 1.40 \\
\hline 1466.13 & OVI(1038) & 0.41298 & 0.608 & 0.059 & 10.34 & \\
\hline 1380.00 & CIII(977) & 0.41246 & 0.691 & 0.060 & 11.51 & \\
\hline 1397.89 & NIII(989) & 0.41230 & 0.153 & 0.060 & 2.55 & \\
\hline 1704.29 & SiIII(1206) & 0.41259 & 0.809 & 0.040 & 20.23 & \\
\hline \multirow[t]{4}{*}{1750.49} & $\mathrm{NV}(1238)$ & 0.41303 & 0.295 & 0.030 & 9.83 & \\
\hline & $\mathrm{NV}(1242)$ & & $\leq 0.06^{\mathrm{h}}$ & & & \\
\hline & $\operatorname{CIV}(1548)$ & & $\leq 0.18^{\mathrm{h}}$ & & & \\
\hline & CIV(1550) & & $\leq 0.18^{\mathrm{h}}$ & & & \\
\hline
\end{tabular}


Table 4-Continued

\begin{tabular}{|c|c|c|c|c|c|c|}
\hline $\begin{array}{l}\text { Wavelength } \\
(\AA)\end{array}$ & ID & $z_{o b s}$ & $\begin{array}{c}W_{o b s} \\
(\AA)\end{array}$ & $\begin{array}{c}\sigma(W) \\
(\AA)\end{array}$ & $\mathrm{SL}^{\mathrm{b}}$ & $\mathrm{LR}^{\mathrm{c}}$ \\
\hline & \multicolumn{2}{|c|}{$1206+4557$} & \multicolumn{2}{|c|}{$z_{e m}=1.158$} & \multicolumn{2}{|l|}{$\mathrm{V}=15.79^{\mathrm{d}}$} \\
\hline 4 & \multicolumn{6}{|c|}{$z_{\text {ave }}=0.73385, \sigma_{z}=0.00032 \mathrm{~A}^{\mathrm{e}}$} \\
\hline 2107.78 & $\operatorname{Ly} \alpha$ & 0.73384 & 0.847 & 0.051 & 16.54 & 0.75 \\
\hline 1779.34 & $\operatorname{Ly} \beta$ & 0.73472 & $1.131^{\mathrm{i}}$ & 0.081 & 13.96 & \\
\hline 1789.01 & OVI(1032) & 0.73365 & 0.279 & 0.077 & 3.62 & 1.15 \\
\hline \multirow[t]{3}{*}{1799.50} & OVI(1038) & 0.73426 & 0.243 & 0.076 & 3.19 & \\
\hline & $\mathrm{NV}(1238)$ & & $\leq 0.44^{\mathrm{h}}$ & & & \\
\hline & NV(1242) & & $\leq 0.44^{\mathrm{h}}$ & & & \\
\hline 2684.95 & $\operatorname{CIV}(1548)$ & 0.73424 & 0.371 & 0.066 & 5.62 & \\
\hline & CIV (1550) & & $\leq 0.13^{\mathrm{h}}$ & & & \\
\hline 5 & \multicolumn{6}{|c|}{$z_{a v e}=0.92703, \sigma_{z}=0.00042 \mathrm{~A}, \mathrm{~B}, \mathrm{C}^{\mathrm{e}}$} \\
\hline 2342.55 & $\operatorname{Ly} \alpha$ & 0.92696 & 4.490 & $0.122^{\mathrm{f}}$ & 36.80 & 1.50 \\
\hline 1976.11 & $\operatorname{Ly} \beta$ & 0.92656 & 3.001 & $0.068^{\mathrm{f}}$ & 43.87 & 2.27 \\
\hline 1874.54 & $\mathrm{Ly} \gamma$ & 0.92747 & 1.320 & $0.076^{\mathrm{f}}$ & 17.32 & \\
\hline 1987.73 & OVI(1032) & 0.92623 & 2.328 & 0.067 & 34.75 & 0.94 \\
\hline 1999.29 & OVI(1038) & 0.92680 & 2.490 & 0.067 & 37.16 & \\
\hline 1881.66 & CIII(977) & 0.92592 & $4.066^{\mathrm{j}}$ & 0.076 & 53.50 & \\
\hline 1907.79 & NIII(989) & 0.92745 & 1.113 & 0.078 & 14.27 & \\
\hline 2294.61 & $\operatorname{SiII}(1190)$ & 0.92757 & 0.295 & 0.046 & 6.41 & \\
\hline 2300.26 & $\operatorname{SiII}(1193)$ & 0.92766 & 0.488 & 0.046 & 10.61 & \\
\hline 2326.10 & SiIII(1206) & 0.92797 & 1.379 & 0.124 & 11.12 & \\
\hline 2386.94 & NV(1238) & 0.92679 & 1.792 & 0.117 & 15.32 & 1.64 \\
\hline 2394.23 & NV(1242) & 0.92648 & 1.093 & 0.117 & 9.34 & \\
\hline 2573.14 & CII(1334) & 0.92812 & 1.835 & 0.075 & 24.47 & \\
\hline 2686.56 & $\operatorname{SiIV}(1393)$ & 0.92757 & 1.294 & 0.067 & 19.31 & 1.20 \\
\hline 2703.75 & $\operatorname{SiIV}(1402)$ & 0.92743 & 1.079 & 0.067 & 16.10 & \\
\hline 2984.04 & CIV(1548) & 0.92742 & 4.440 & 0.096 & 46.25 & 1.62 \\
\hline 2988.88 & CIV(1550) & 0.92735 & 2.737 & 0.096 & 28.51 & \\
\hline \multicolumn{7}{|l|}{6} \\
\hline 2529.96 & $\operatorname{Ly} \alpha$ & 1.08113 & 1.243 & 0.105 & 11.84 & 0.98 \\
\hline 2134.35 & $\operatorname{Ly} \beta$ & 1.08298 & 1.262 & $0.046^{\mathrm{f}}$ & 27.43 & 2.69 \\
\hline 2023.47 & $\mathrm{Ly} \gamma$ & 1.08060 & 0.469 & 0.057 & 8.23 & \\
\hline 2149.35 & OVI(1032) & 1.08284 & 2.543 & $0.044^{\mathrm{f}}$ & 58.06 & 1.18 \\
\hline 2161.21 & OVI(1038) & 1.08285 & 2.160 & $0.044^{\mathrm{f}}$ & 49.54 & \\
\hline \multirow[t]{3}{*}{2510.57} & SiIII(1206) & 1.08087 & 1.038 & 0.112 & 9.27 & \\
\hline & $\mathrm{NV}(1238)$ & & $\leq 0.17^{\mathrm{h}}$ & & & \\
\hline & $\mathrm{NV}(1242)$ & & $\leq 0.17^{\mathrm{h}}$ & & & \\
\hline 3224.76 & CIV(1548) & 1.08291 & 0.605 & 0.101 & 5.98 & 1.20 \\
\hline 3229.31 & $\operatorname{CIV}(1550)$ & 1.08239 & 0.505 & 0.101 & 5.00 & \\
\hline
\end{tabular}


Table 4-Continued

\begin{tabular}{|c|c|c|c|c|c|c|}
\hline $\begin{array}{l}\text { Wavelength } \\
\qquad(\AA)\end{array}$ & ID & $z_{o b s}$ & $\begin{array}{c}W_{o b s} \\
(\AA)\end{array}$ & $\begin{array}{c}\sigma(W) \\
(\AA)\end{array}$ & $\mathrm{SL}^{\mathrm{b}}$ & $\mathrm{LR}^{\mathrm{c}}$ \\
\hline & \multicolumn{2}{|c|}{$1317+2743$} & \multicolumn{2}{|c|}{$z_{e m}=1.022$} & \multicolumn{2}{|l|}{$\mathrm{V}=15.98^{\mathrm{d}}$} \\
\hline \multicolumn{7}{|c|}{ No O VI Detected } \\
\hline & \multicolumn{2}{|c|}{$1352+0106$} & \multicolumn{2}{|c|}{$z_{e m}=1.121$} & \multicolumn{2}{|l|}{$\mathrm{V}=16.03^{\mathrm{d}}$} \\
\hline 7 & \multicolumn{5}{|c|}{$z_{a v e}=0.66752, \sigma_{z}=0.00034 \mathrm{~A}, \mathrm{~B}, \mathrm{C}^{\mathrm{e}}$} & \\
\hline 2026.88 & $\operatorname{Ly} \alpha$ & 0.66730 & 2.920 & $0.065^{\mathrm{f}}$ & 45.06 & 1.34 \\
\hline 1710.59 & $\operatorname{Ly} \beta$ & 0.66769 & 2.177 & $0.086^{\mathrm{f}}$ & 25.20 & \\
\hline 1721.07 & OVI(1032) & 0.66782 & 1.089 & 0.084 & 12.90 & 2.46 \\
\hline 1730.69 & OVI(1038) & 0.66794 & 0.442 & 0.083 & 5.35 & \\
\hline 1985.62 & $\operatorname{SiII}(1190)$ & 0.66800 & 0.503 & 0.064 & 7.86 & \\
\hline 1989.50 & $\operatorname{SIII}(1193)$ & 0.66724 & 1.084 & 0.064 & 16.94 & \\
\hline 2011.92 & $\operatorname{SiIII}(1206)$ & 0.66757 & 1.652 & $0.065^{\mathrm{f}}$ & 25.41 & \\
\hline 2066.08 & $\mathrm{NV}(1238)$ & 0.66778 & 0.485 & 0.053 & 9.15 & 1.49 \\
\hline 2072.16 & NV(1242) & 0.66733 & 0.325 & 0.052 & 6.25 & \\
\hline 2225.39 & CII(1334) & 0.66754 & 1.197 & 0.047 & 25.47 & \\
\hline 2324.18 & $\operatorname{SiIV}(1393)$ & 0.66757 & 1.225 & 0.124 & 9.88 & 0.73 \\
\hline 2338.93 & $\operatorname{SiIV}(1402)$ & 0.66737 & 1.681 & 0.123 & 13.67 & \\
\hline 2581.02 & CIV(1548) & 0.66711 & 2.219 & 0.074 & 29.98 & 1.16 \\
\hline \multirow[t]{2}{*}{2585.92} & CIV(1550) & 0.66750 & 1.921 & 0.074 & 25.96 & \\
\hline & \multicolumn{2}{|c|}{$1407+2632$} & \multicolumn{2}{|c|}{$z_{e m}=0.944$} & \multicolumn{2}{|l|}{$\mathrm{V}=15.73^{\mathrm{d}}$} \\
\hline \multicolumn{7}{|c|}{ No O VI Detected } \\
\hline & \multicolumn{2}{|c|}{$1424-1150$} & \multicolumn{2}{|c|}{$z_{e m}=0.806$} & $\mathrm{~V}=16.49^{\mathrm{d}}$ & \\
\hline 8 & \multicolumn{5}{|c|}{$z_{\text {ave }}=0.65516, \sigma_{z}=0.00027 \mathrm{~A}, \mathrm{~B}^{\mathrm{e}}$} & \\
\hline 2012.11 & $\operatorname{Ly} \alpha$ & 0.65514 & 1.959 & $0.083^{f}$ & 23.72 & 1.58 \\
\hline 1697.85 & $\operatorname{Ly} \beta$ & 0.65528 & 1.237 & $0.101^{\mathrm{f}}$ & 12.22 & \\
\hline 1707.88 & OVI(1032) & 0.65503 & 0.313 & 0.100 & 3.14 & 0.77 \\
\hline 1716.98 & OVI(1038) & 0.65473 & 0.409 & 0.098 & 4.16 & \\
\hline \multirow[t]{3}{*}{1997.45} & $\operatorname{SiIII}(1206)$ & 0.65557 & 0.299 & 0.083 & 3.60 & \\
\hline & $\operatorname{NV}(1238)$ & & $\leq 0.17^{\mathrm{h}}$ & & & \\
\hline & $\mathrm{NV}(1242)$ & & $\leq 0.17^{\mathrm{h}}$ & & & \\
\hline 2562.81 & CIV(1548) & 0.65535 & 0.653 & 0.137 & 4.77 & 0.78 \\
\hline 2567.06 & $\operatorname{CIV}(1550)$ & 0.65535 & 0.842 & 0.137 & 6.15 & \\
\hline \multicolumn{7}{|c|}{$z_{\text {ave }}=0.74654, \sigma_{z}=0.00042 \mathrm{~A}, \mathrm{~B}^{\mathrm{e}}$} \\
\hline 2123.49 & $\operatorname{Ly} \alpha$ & 0.74676 & 0.463 & 0.062 & 7.42 & $\geq 2.80$ \\
\hline 1801.81 & OVI(1032) & 0.74606 & 0.542 & 0.083 & 6.56 & 1.91 \\
\hline \multirow[t]{5}{*}{1812.53} & OVI(1038) & 0.74682 & 0.284 & 0.081 & 3.51 & \\
\hline & $\mathrm{NV}(1238)$ & & $\leq 0.11^{\mathrm{h}}$ & & & \\
\hline & $\mathrm{NV}(1242)$ & & $\leq 0.11^{\mathrm{h}}$ & & & \\
\hline & CIV(1548) & & $\leq 0.22^{\mathrm{h}}$ & & & \\
\hline & $\operatorname{CIV}(1550)$ & & $\leq 0.22^{\mathrm{h}}$ & & & \\
\hline
\end{tabular}


Table 4-Continued

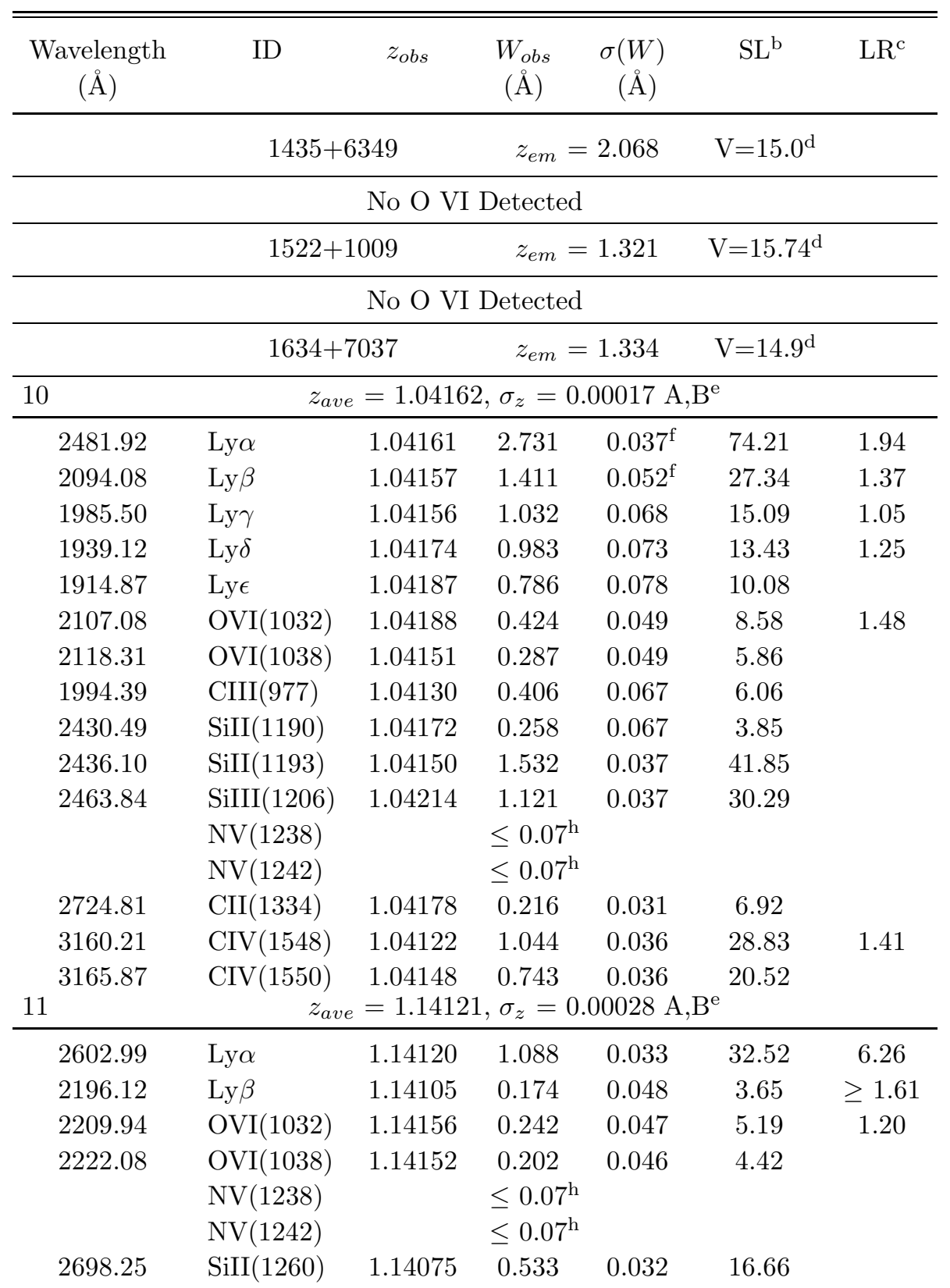


Table 4-Continued

\begin{tabular}{|c|c|c|c|c|c|c|}
\hline $\begin{array}{c}\text { Wavelength } \\
(\AA)\end{array}$ & ID & $z_{o b s}$ & $\begin{array}{c}W_{o b s} \\
(\AA)\end{array}$ & $\begin{array}{c}\sigma(W) \\
(\AA)\end{array}$ & $\mathrm{SL}^{\mathrm{b}}$ & $\mathrm{LR}^{\mathrm{c}}$ \\
\hline & \multicolumn{2}{|c|}{$2340-0340$} & \multicolumn{2}{|c|}{$z_{e m}=0.896$} & $\mathrm{~V}=16.02^{\mathrm{d}}$ & \\
\hline 12 & \multicolumn{5}{|c|}{$z_{\text {ave }}=0.68416, \sigma_{z}=0.00029 \mathrm{~A}, \mathrm{~B}^{\mathrm{e}}$} & \\
\hline 2047.22 & $\operatorname{Ly} \alpha$ & 0.68403 & 1.764 & 0.065 & 26.97 & $0.94^{\mathrm{i}}$ \\
\hline 1727.90 & $\operatorname{Ly} \beta$ & 0.68457 & 1.882 & 0.094 & 19.94 & $5.88^{\mathrm{i}}$ \\
\hline 1637.57 & $\mathrm{Ly} \gamma$ & 0.68381 & 0.320 & 0.134 & 2.39 & \\
\hline 1737.92 & OVI(1032) & 0.68415 & 0.525 & 0.093 & 5.62 & 1.68 \\
\hline 1747.65 & OVI(1038) & 0.68428 & 0.312 & 0.092 & 3.39 & \\
\hline 1645.67 & $\operatorname{CIII}(977)$ & 0.68438 & 1.657 & 0.127 & 13.05 & \\
\hline 2031.65 & $\operatorname{SiIII}(1206)$ & 0.68392 & 0.466 & 0.067 & 6.96 & \\
\hline \multirow[t]{2}{*}{2086.02} & $\mathrm{NV}(1238)$ & 0.68388 & 0.148 & 0.062 & 2.39 & $\geq 1.19$ \\
\hline & $\mathrm{NV}(1242)$ & & $\leq 0.12^{\mathrm{h}}$ & & & \\
\hline 2247.02 & CII(1334) & 0.68375 & 0.201 & 0.044 & 4.57 & \\
\hline
\end{tabular}

a Table contains 11 Objects, 20 Spectra, \& 12 O VI detections.

b Significance Level: $W_{o b s} / \sigma(W)$.

${ }^{\mathrm{c}}$ Line Ratio: Ratio of equivalent widths is calculated for the following pairs of lines. (Ly $\alpha / \mathrm{Ly} \beta, \mathrm{Ly} \beta / \mathrm{Ly} \gamma, \mathrm{O} \mathrm{VI}(1032) / \mathrm{O} \mathrm{VI}(1038), \mathrm{N}$ V(1238)/N V(1242), $\mathrm{C}$ IV(1548)/C IV(1550)). Ratio is listed with stronger line. If weaker line is not detected above the $2 \sigma$ equivalent width limit, then that limit is used in the Line Ratio.

d Visual Magnitude

e $z_{\text {ave }}$ is calculated as the weighted mean of $\operatorname{Ly} \alpha, \operatorname{Ly} \beta, \operatorname{Ly} \gamma, \mathrm{O} \operatorname{VI}(1032)$ \& O VI(1038). See text for a desciption of the weighting procedure. Standard deviation is calculated for the five lines above. The samples in which the system has been included are marked by the letters A,B,C.

$\mathrm{f}$ This line had an upper limit, described in the text, used in place of its Significance Level as the weight for calculating $z_{\text {ave }}$.

g $\mathrm{O} \mathrm{VI}(1032)$ at $z_{a b s}=0.9534$ is blended with $\operatorname{Ly} \beta$ at $z_{a b s}=0.9669$.

h Absorption due to this ion is not detected at the $2 \sigma$ level at $z_{\text {ave }}$. An upper limit equal to the $2 \sigma$ equivalent width level is listed.

${ }^{i}$ The line ratio does not fall within the $1 \sigma$ error of the theoretical prediction.

$\mathrm{j}$ This line is blended producing excess equivalent width. 


\begin{tabular}{ccccccc} 
QSO & $z_{a b s}$ & \multicolumn{3}{c}{ Rest Equivalent Widths $(\AA)$} & \multicolumn{2}{c}{$\begin{array}{c}\text { Doublet Ratio } \\
\text { O V V }\end{array}$} \\
& & O VI & C II & Subtracted $^{b}$ & Fitted $^{\text {c }}$ \\
& & 1032 & $1036-8^{\mathrm{a}}$ & 1334 & & \\
\hline $0122-0021$ & 0.9534 & 0.62 & 0.44 & 0.18 & $2.00^{\mathrm{d}}$ & 1.40 \\
& 0.9669 & 0.36 & 0.25 & $\leq 0.16$ & 1.44 & 1.44 \\
$0454-2203$ & 0.4126 & 0.60 & 0.43 & $\leq 0.02$ & 1.40 & 1.40 \\
$1206+4557$ & 0.7339 & 0.16 & 0.14 & $\leq 0.11$ & 1.14 & 1.15 \\
& 0.9270 & 1.21 & 1.29 & $\leq 0.11$ & 0.94 & 0.94 \\
& 1.0828 & 1.22 & 1.04 & $\leq 0.10$ & 1.17 & 1.18 \\
$1352+0106$ & 0.6675 & 0.65 & 0.85 & 0.72 & $1.98^{\mathrm{d}}$ & 2.46 \\
$1424-1150$ & 0.6552 & 0.19 & 0.25 & $\leq 0.09$ & 0.76 & 0.76 \\
& 0.7465 & 0.31 & 0.16 & $\leq 0.17$ & 1.94 & 1.91 \\
$1634+7037$ & 1.0416 & 0.21 & 0.17 & 0.10 & $1.96^{\mathrm{d}}$ & 1.48 \\
& 1.1412 & 0.11 & 0.09 & $\leq 0.03$ & 1.22 & 1.20 \\
$2340-0340$ & 0.6842 & 0.31 & 0.35 & 0.12 & $1.37^{\mathrm{d}}$ & 1.68
\end{tabular}

Table 5: Doublet Ratios

${ }^{a}$ Rest equivalent width including both $\mathrm{O}$ VI(1038) and C II(1036).

${ }^{b} \mathrm{~W}(1032) / \mathrm{W}(1038)$, corrected for C II(1036) when C II(1334) was detected.

${ }^{c} \mathrm{~W}(1032) / \mathrm{W}(1038)$, using W for O VI (1038) deblended from C II(1036) in Table 4.

${ }^{d}$ Doublet ratio corrected for calculated C II(1036). 


\begin{tabular}{|c|c|c|c|c|c|c|c|}
\hline \multirow[t]{3}{*}{ QSO } & \multirow[t]{3}{*}{$z_{a b s}$} & \multicolumn{2}{|c|}{ Oxygen $\mathrm{VI}^{\mathrm{b}}$} & \multicolumn{2}{|c|}{ Carbon IV $^{\mathrm{c}}$} & \multicolumn{2}{|c|}{ Hydrogen $\mathrm{I}^{\mathrm{d}}$} \\
\hline & & $\log (\mathrm{N})$ & b & $\log (\mathrm{N})$ & b & $\log (\mathrm{N})$ & $\mathrm{b}$ \\
\hline & & $\mathrm{cm}^{-2}$ & $\mathrm{~km} \mathrm{~s}^{-1}$ & $\mathrm{~cm}^{-2}$ & $\mathrm{~km} \mathrm{~s}^{-1}$ & $\mathrm{~cm}^{-2}$ & $\mathrm{~km} \mathrm{~s}^{-1}$ \\
\hline \multirow[t]{2}{*}{ 0122-0021 } & 0.9534 & $15.1_{-0.3}^{+0.3}$ & $74_{-25}^{+70}$ & $15.3_{-0.5}^{+2.3}$ & $54_{-20}^{+28}$ & $17.0_{-0.5}^{+1.5}$ & $53_{-13}^{+10}$ \\
\hline & 0.9669 & $14.8_{-0.2}^{+1.3}$ & $45_{-26}^{+100}$ & $13.9^{\mathrm{e}}$ & $\ldots$ & $16.1_{-0.2}^{+0.4}$ & $49_{-9}^{+8}$ \\
\hline 0454-2203 & 0.4126 & $15.1_{-0.2}^{+0.2}$ & $70_{-20}^{+39}$ & $\leq 13.5^{\mathrm{g}}$ & $\ldots$ & $\ldots \mathrm{f}$ & $\ldots \mathrm{f}$ \\
\hline \multirow[t]{3}{*}{$1206+4557$} & 0.7339 & & $\ldots \mathrm{f}$ & $13.7^{\mathrm{e}}$ & $\ldots$ & $\ldots$ & \\
\hline & 0.9270 & $\ldots \mathrm{f}$ & $\ldots \mathrm{f}$ & $15.0_{-0.1}^{+0.1}$ & $223_{-1}^{+45}$ & $18.7_{-0.7}^{+0.9}$ & $86_{-10}^{+9}$ \\
\hline & 1.0828 & $15.7_{-0.1}^{+0.2}$ & $106_{-10}^{+10}$ & $\ldots \mathrm{f}$ & $\ldots \mathrm{f}$ & & \\
\hline $1352+0106$ & 0.6675 & $14.7^{\mathrm{e}}$ & $\ldots$ & $15.3_{-0.2}^{+0.7}$ & $74_{-18}^{+13}$ & $\ldots \mathrm{f}$ & \\
\hline \multirow[t]{2}{*}{$1424-1150$} & 0.6552 & $\ldots{ }^{f}$ & $\ldots \mathrm{f}$ & $\ldots \mathrm{f}$ & $\ldots \mathrm{f}$ & $17.8_{-0.9}^{+2.0}$ & $49_{-16}^{+6}$ \\
\hline & 0.7465 & $14.4^{\mathrm{e}}$ & $\ldots$ & $\leq 13.5^{\mathrm{g}}$ & $\ldots$ & $13.7^{\mathrm{e}}$ & \\
\hline \multirow[t]{2}{*}{$1634+7037$} & 1.0416 & $14.8_{-0.5}^{+1.3}$ & $20_{-10}^{+50}$ & $14.5_{-0.1}^{+0.1}$ & $40_{-7}^{+11}$ & $16.6_{-0.1}^{+0.2}$ & $63_{-3}^{+3}$ \\
\hline & 1.1412 & $14.6_{-0.5}^{+1.5}$ & $10_{-4}^{+30}$ & $\ldots$ & $\cdots$ & $15.1_{-0.2}^{+0.1}$ & $31_{-3}^{+3}$ \\
\hline $2340-0340$ & 0.6842 & $14.6_{-0.2}^{+1.4}$ & $45_{-32}^{+\infty}$ & $\cdots$ & $\ldots$ & $\ldots \mathrm{f}$ & $\ldots f$ \\
\hline
\end{tabular}

Table 6: Column Densities and Dispersion Velocities ${ }^{\mathrm{a}}$

\footnotetext{
${ }^{a}$ Values are calculated with two equivalent width measurements and their $1 \sigma$ errors.

${ }^{b}$ From lines $\lambda \lambda 1032,1038$.

${ }^{c}$ From lines $\lambda \lambda 1548,1550$.

${ }^{d}$ From lines $\lambda 1215, \lambda 1025$.

${ }^{e}$ Doublet ratio suggests linear absorption. Column density estimate is based on the stronger member.

${ }^{f}$ Absorption lines are too saturated to be effectively analyzed in with the doublet ratio method.

${ }^{g}$ Neither line was detected above $2 \sigma$. Upper limit is placed on column density assuming linear absorption.
} 


\begin{tabular}{ccccccccc} 
Object & $z_{a b s}$ & $\mathrm{~W}(1032)$ & $\mathrm{W}(1025)$ & $R_{O B}$ & $\sigma_{R_{O B}}$ & $\mathrm{~W}(1548)$ & $R_{O C}$ & $\sigma_{R_{O C}}$ \\
\hline $0122-0021$ & 0.9534 & 1.21 & 1.37 & 0.88 & 0.10 & 1.99 & 0.56 & 0.07 \\
& 0.9669 & 0.71 & 0.80 & 0.89 & 0.16 & 0.63 & 1.13 & 0.36 \\
$0454-2203$ & 0.4126 & 0.85 & 0.68 & 1.25 & 0.14 & $\leq 0.07^{\mathrm{a}}$ & $\geq 12.1$ & $\ldots$ \\
$1206+4557$ & 0.7339 & 0.28 & $\leq 0.85^{\mathrm{b}}$ & 0.33 & 0.09 & 0.37 & 0.76 & 0.25 \\
& 0.9270 & 2.32 & 3.00 & 0.77 & 0.03 & 4.44 & 0.53 & 0.02 \\
& 1.0828 & 2.54 & 1.26 & 2.02 & 0.08 & 0.61 & 4.17 & 0.71 \\
$1352+0106$ & 0.6675 & 1.09 & 2.18 & 0.50 & 0.05 & 2.22 & 0.49 & 0.04 \\
$1424-1150$ & 0.6552 & 0.31 & 1.24 & 0.25 & 0.08 & 0.65 & 0.48 & 0.19 \\
& 0.7465 & 0.54 & $\leq 0.17^{\mathrm{c}}$ & $\geq 3.18$ & $\ldots$ & $\leq 0.27^{\mathrm{a}}$ & $\geq 2.00$ & $\ldots$ \\
$1634+7037$ & 1.0416 & 0.42 & 1.41 & 0.33 & 0.04 & 1.04 & 0.40 & 0.05 \\
& 1.1412 & 0.24 & 0.17 & 1.41 & 0.48 & $\leq 0.07^{\mathrm{a}}$ & $\geq 3.4$ & $\ldots$ \\
$2340-0340$ & 0.6842 & 0.53 & 1.88 & 0.28 & 0.08 & $\leq 0.32^{\mathrm{a}}$ & $\geq 1.7$ & $\ldots$
\end{tabular}

Table 7: Ion Ratios

${ }^{a} \mathrm{C} \mathrm{IV(1548)} \mathrm{is} \mathrm{not} \mathrm{detected,} \mathrm{an} \mathrm{upper} \mathrm{limit} 2 \sigma$ equivalent width is listed.

${ }^{b} \mathrm{Ly} \beta$ is apparently blended with another Ly $\alpha$ line; an upper limit of the equivalent width is given by its corresponding $\operatorname{Ly} \alpha$.

${ }^{c} \mathrm{Ly} \beta$ is not detected, an upper limit $2 \sigma$ equivalent width is listed. 


\section{REFERENCES}

Anders, E., \& Grevesse, N. 1989, Geochim. Cosmochim. Acta, 53, 197

Bahcall, J. N., Bergeron, J., Boksenberg, A., Hartig, G. F., Jannuzi, B. T., Kirhakos, S., Sargent, W. L. W., Savage, B. D., Schneider, D. P., Turnshek, D. A., Weymann, R. J., \& Wolfe, A. 1993, ApJS, 87, 1

Barcons, X., Fabian A. C., \& Rees, M. J. 1991, Nature, 350, 685

Bergeron, J., Petitjean, P., Sargent, W. L. W., Bahcall, J. N., Boksenberg, A., Hartig, G. F., Jannuzi, B. T., Kirhakos, S., Savage, B. D., Schneider, D. P., Turnshek, D. A., Weymann, R. J., \& Wolfe, A. 1994, ApJ, 436, 33

Bergeron, J. 1988, in QSO Absorption Lines: Probing the Universe, ed. J. C. Blades, D. A. Turnshek, \& C. A. Norman (Cambridge: Cambridge Univ. Press), 127

Burrows, D. N., \& Mendenhall, J. A. 1991, Nature, 351, 629

Cohen, R. D., Bartko, F., Beaver, E. A., Burbidge, E. M., Junkkarinen, V. T., Lyons, R. W., Rosenblatt, E. I., Burks, G. S., Harms, R. J., \& Henriksen, M. 1991, BAAS, 231426

Cox, D. P., \& Tucker, W. H. 1969, ApJ, 157, 1157

Davidsen, A. F., Bowers, C. W., Kruk, J. W., Kriss, G. A., Ferguson, H. C., Blair, W. P., Kimble, R. A., \& Long, K. S. 1991, BAAS, 23, 1472

Fabbiano, G. 1989, ARA\&A, 27, 87

Fan, L., \& Ikeuchi, S. 1992, ApJ, 390, 405

Fan, X.-M. 1995, Ph.D. Thesis, Columbia University

Forman, W., Schwarz, J., Jones, C., Liller, W., \& Fabian, A. C. 1979, ApJL, 234, L27

Gnedin, N. Y., \& Ostriker, J. P. 1992 ApJ 400, 1

Hamann, F., Barlow, T. A., Beaver, E. A., Burbidge, E. M., Cohen, R. D., Junkkarinen, V., \& Lyons, R. 1995, ApJ, 443, 606

Hamann, F., Zuo, L., \& Tytler, D. 1995, Submitted to ApJ

Hartquist, T. W., \& Snijders, M. A. J. 1982, Nature, 299, 783

Jakobsen, P., Boksenberg, A., Deharveng, J. M., Greenfield, P., Jedrzejewski, R., \& Paresce, F. 1994, Nature, 370,35

Lanzetta, K. M., Turnshek, D. A., \& Wolfe, A. M. 1987, ApJ, 322, 739

Lanzetta, K. M., Wolfe, A. M., Turnshek, D. A., Lu, L., McMahon, R. G., \& Hazard, C. 1991, ApJS, 77, 1

Lu, L., \& Savage, B. D. 1993, ApJ, 403, 127

Madua, P. 1994, private communication

Morton, D. C. 1991, ApJS, 77, 119

Pettini, M., \& D’Odorico, S. 1986, ApJ, 310, 700

Reimers, D., et al. 1992, Nature, 360, 561 
Sargent, W. L. W., Steidel, C. C., \& Boksenberg A. 1989, ApJS, 69, 703

Sargent, W. L. W., Boksenberg, A., \& Steidel, C. C. 1990, ApJS, 68, 539

Schneider, D. P., Hartig, G. F., Jannuzi, B. T., Kirhakos, S., Saxe, D. H., Weymann, R. J., Bahcall, J. N., Bergeron, J., Boksenberg, A., Sargent, W. L. W., Savage, B. D., Turnshek, D. A., \& Wolfe, A. 1993, ApJS, 87, 45

Sembach, K. R., \& Savage, B.D. 1992, ApJS, 83, 147

Shapiro, P. R., \& Moore, R. T. 1976, ApJ, 207, 460

Shapiro, P. R., \& Benjamin, R. 1991 PASP, 103, 923

Snowden, S. L., Mebold, U., Hirth, W., Herbstmeier, U., \& Schmitt, J. H. M. M. 1991, Science, 252, 1529

Songaila, A., Cowie, L. L., Hogan, C. J., \& Rugers, M. 1994 Nature, 368, 599

Spitzer, L. 1978, Physical processes in the interstellar medium, (New York:Wiley), p.44

Steidel, C. C., 1990, ApJS, 74, 37

Tytler, D., \& Fan, X. M. 1994 BAAS, 27, 1424

Tytler, D., Boksenberg, A., Sargent, W. L. W., Young, P., \& Kunth, D. 1987, ApJS, 64, 667

Verner, D. A., Barthel, P. D., \& Tytler, D. 1994, A\&AS, 108, 287

Verner, D. A., Tytler, D., \& Barthel, P. D. 1994, ApJ, 430, 186 (VTB94)

Verner, D. A., \& Yakolev, D. G. 1990, Ap\&SS, 165, 27

Wang, Q. 1991, ApJL, 377, L85

Wolfe, A. M., Turnshek, D. A., Smith, H. E., \& Cohen, R. D. 1986, ApJS, 61, 249

Wolfe, A. M., Lanzetta, K. M., Foltz, C. B., \& Chaffee, F. H. 1995, ApJ, submitted

Wright, E.L., Mather, J.C., Fixsen, D.J., Kogut, A., Shafer, R. A., Bennett, C. L., Boggess, N. W., Cheng, E. S., Silverberg, R. F., Smoot, G. F., \& Weiss, R. 1994 ApJ, 420, 450

Young, P. J., Sargent, W. L. W., Boksenberg, A., Carswell, R. F., \& Whelan, J. A. J. 1979, ApJ, 229, 891 
Fig. 1.- Number of chance coincidences of new redshift systems with two lines (O VI pairs), three lines ( $\mathrm{Ly} \alpha$ and $\mathrm{O} \mathrm{VI})$, and four lines $(\mathrm{Ly} \alpha, \mathrm{Ly} \beta$ and $\mathrm{O} \mathrm{VI})$. We used a random distribution of lines, and accpeted lines into a system if the redshift for each line fell within $\Delta z=0.0005$ of all the others. We see that the number of false three lined systems is about $10 \%$ that of the two lined systems. Each HST spectrum covers $700-1000 \AA$, the real lines density is 80.0 per $1000 \AA$ An the Lyman- $\alpha$ forest, and real O VI systems occur about 1 per $1000 \AA$ in the rest frame. Therefore, we require at least 3 lines per system in order to avoid excessive coincidences.

Fig. 2.- The spectra of 11 QSOs plotted with the fitted continuum and $1 \sigma$ error. Lines identified with Oxygen VI absorption systems are labeled (see Table 4). Labels of the same height indicate lines of a common system.

Fig. 3.- Plots of Lyman- $\alpha$, Lyman- $\beta$, O VI, and C IV absorption in velocity space for each of the 12 systems in which O VI was identified. Zero velocity corresponds to the $z_{a b s}$ indicated for each system. Flux scales are different depending on the strength of absorption.

Fig. 4.- Calculated O VI Doublet Ratio versus Column Density for a single absorbing cloud. Our search was sensitive to the right of the vertical dashed line at $\log \left(\mathrm{N}_{O V I}\right)>14.5$, which corresponds to $\mathrm{W}(1038)>0.21 \AA$.

Fig. 5.- Equivalent wdith ratios for the 12 systems in Sample A. Ratios of equivalent widths and the $1 \sigma$ error are calculated in each system where absorption was detected at the respective wavelengths. Lower limits are shown with arrows in the systems where C IV or Ly $\beta$ are not detected. 


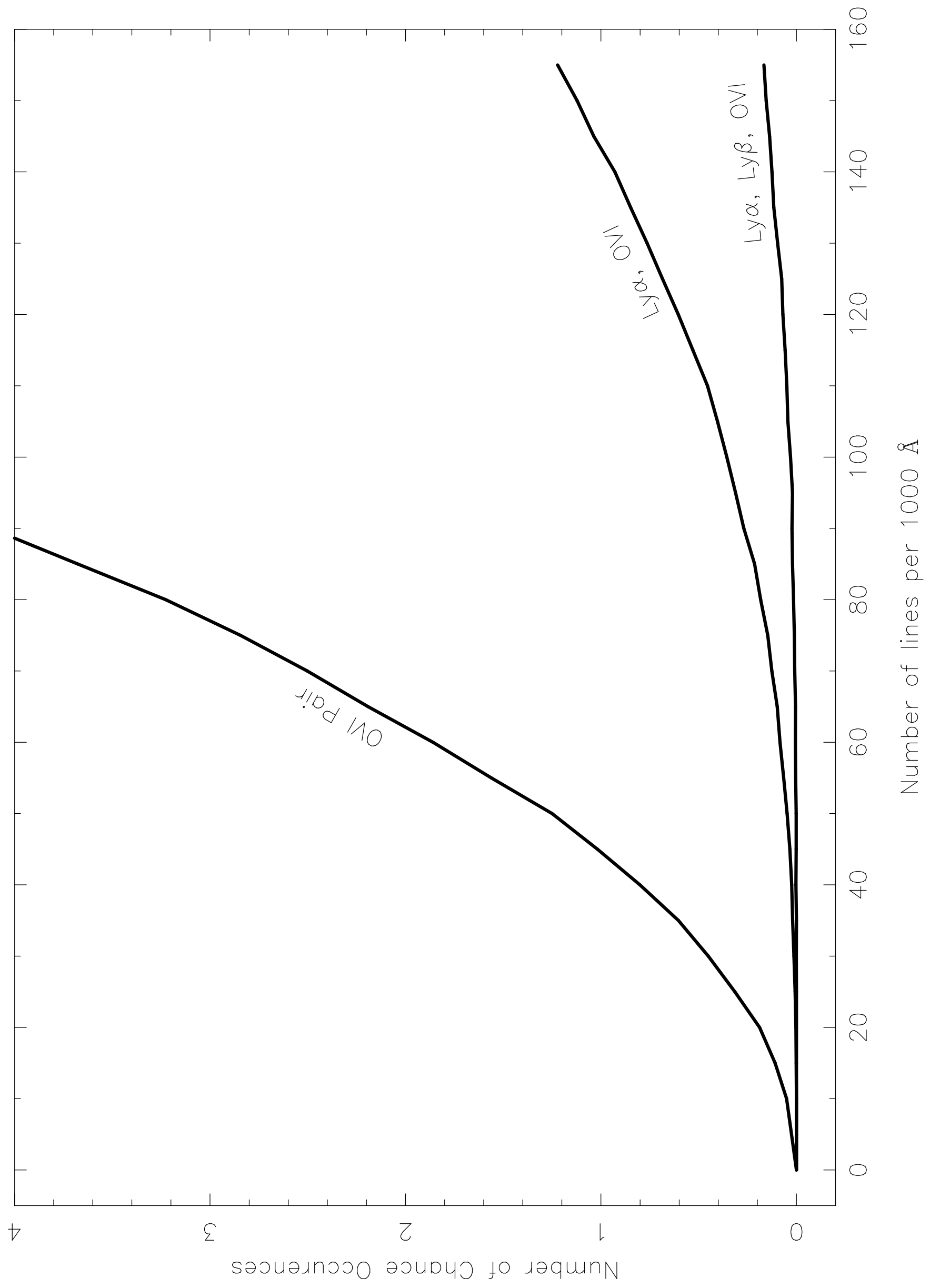




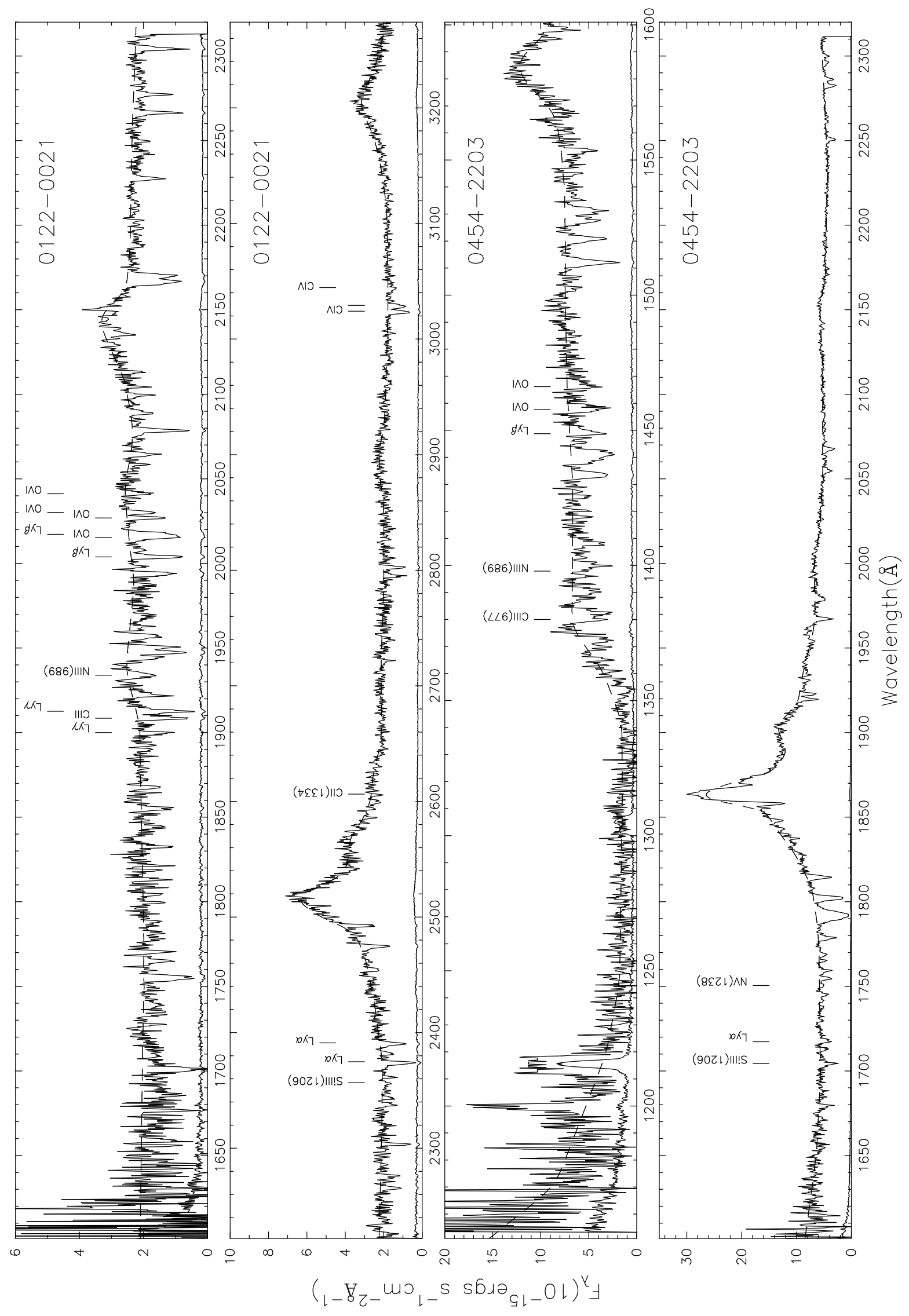




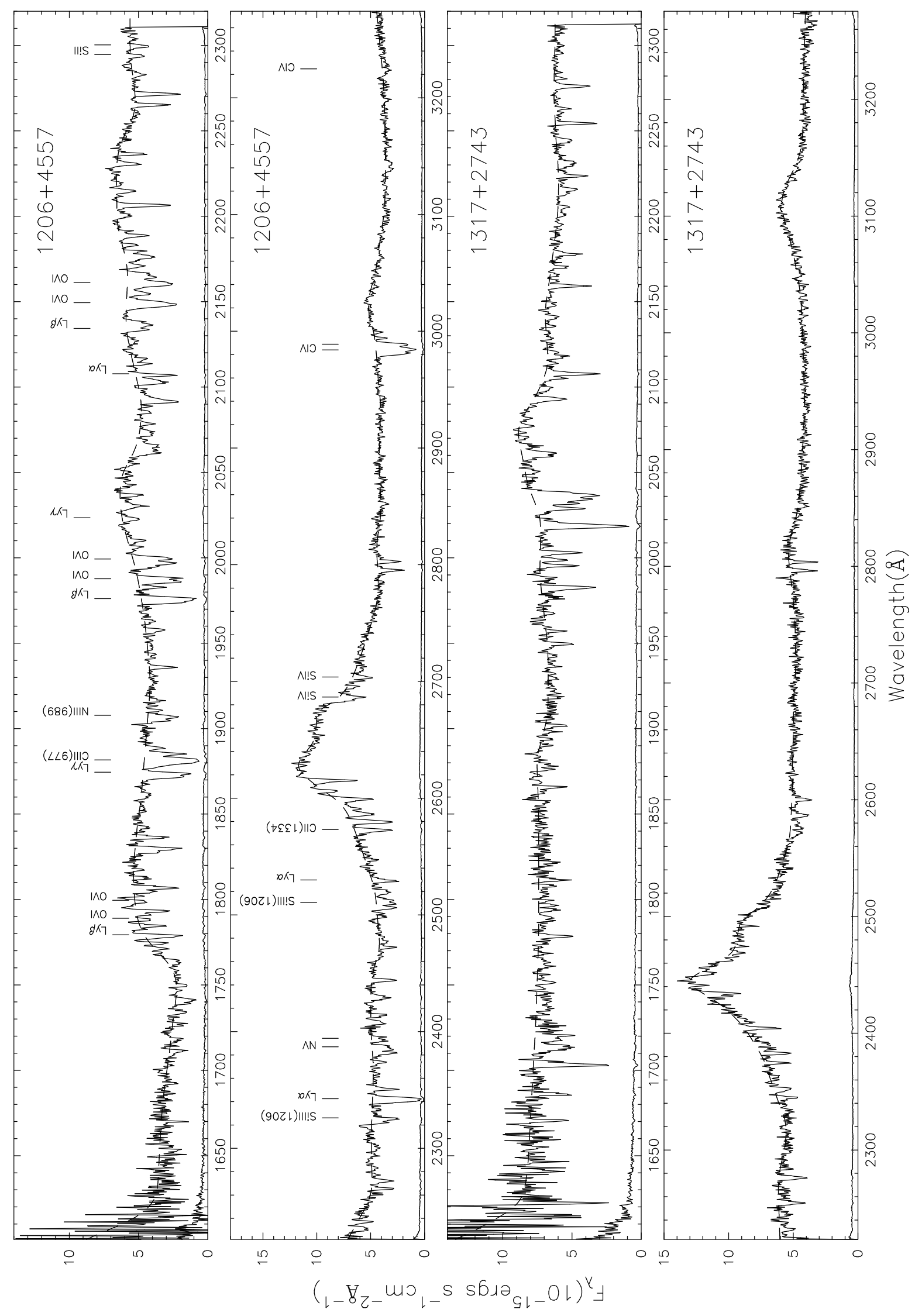




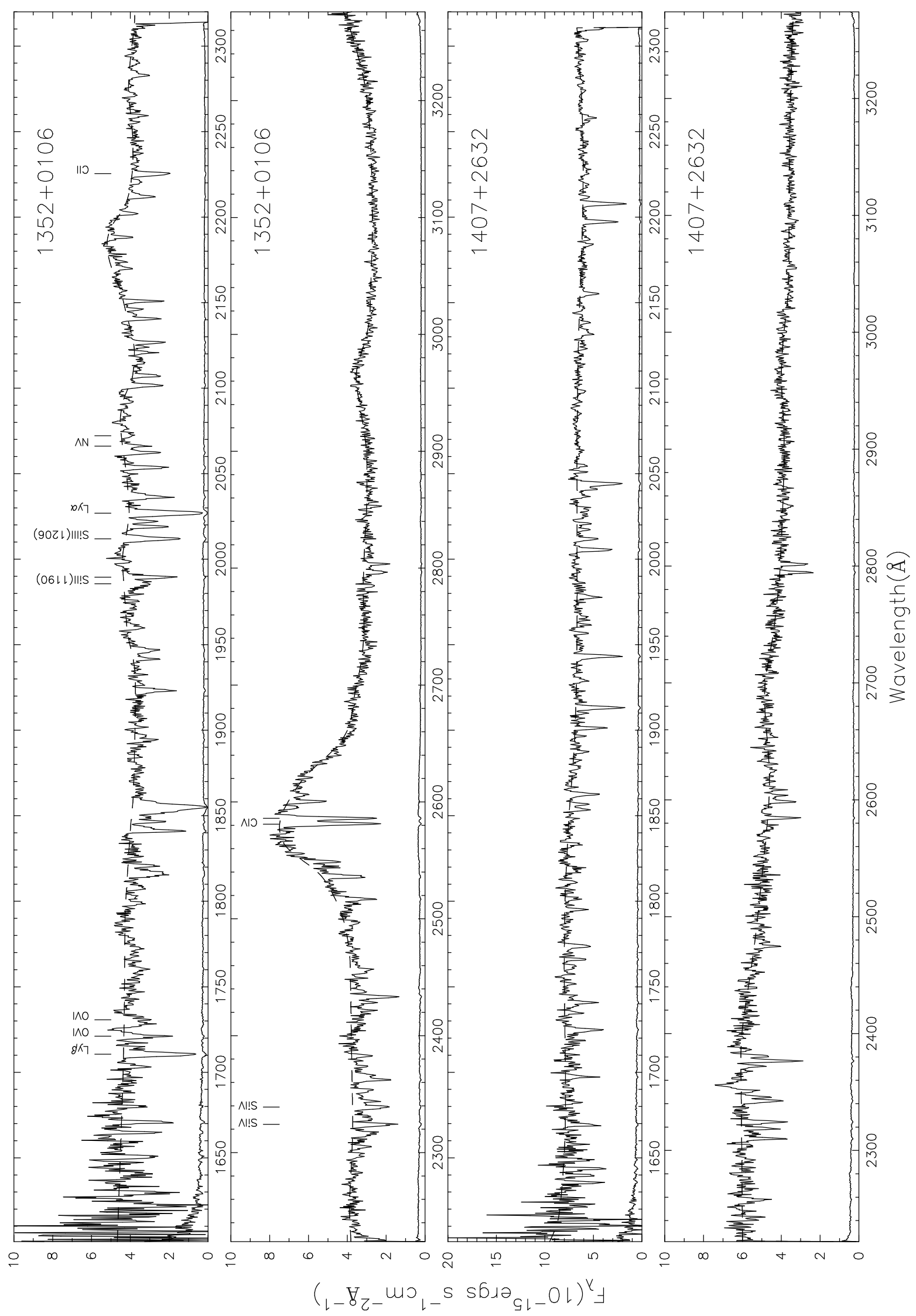




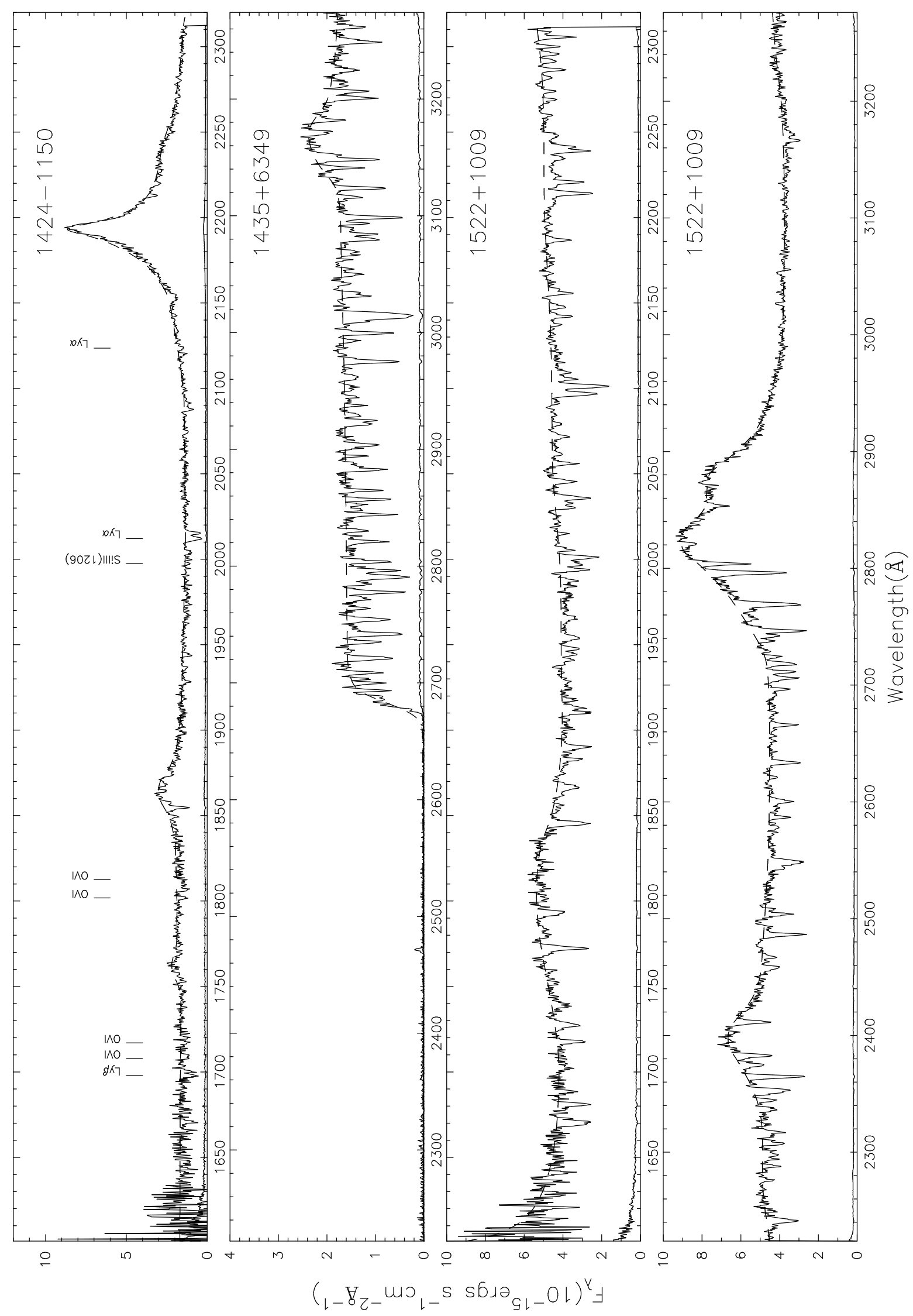




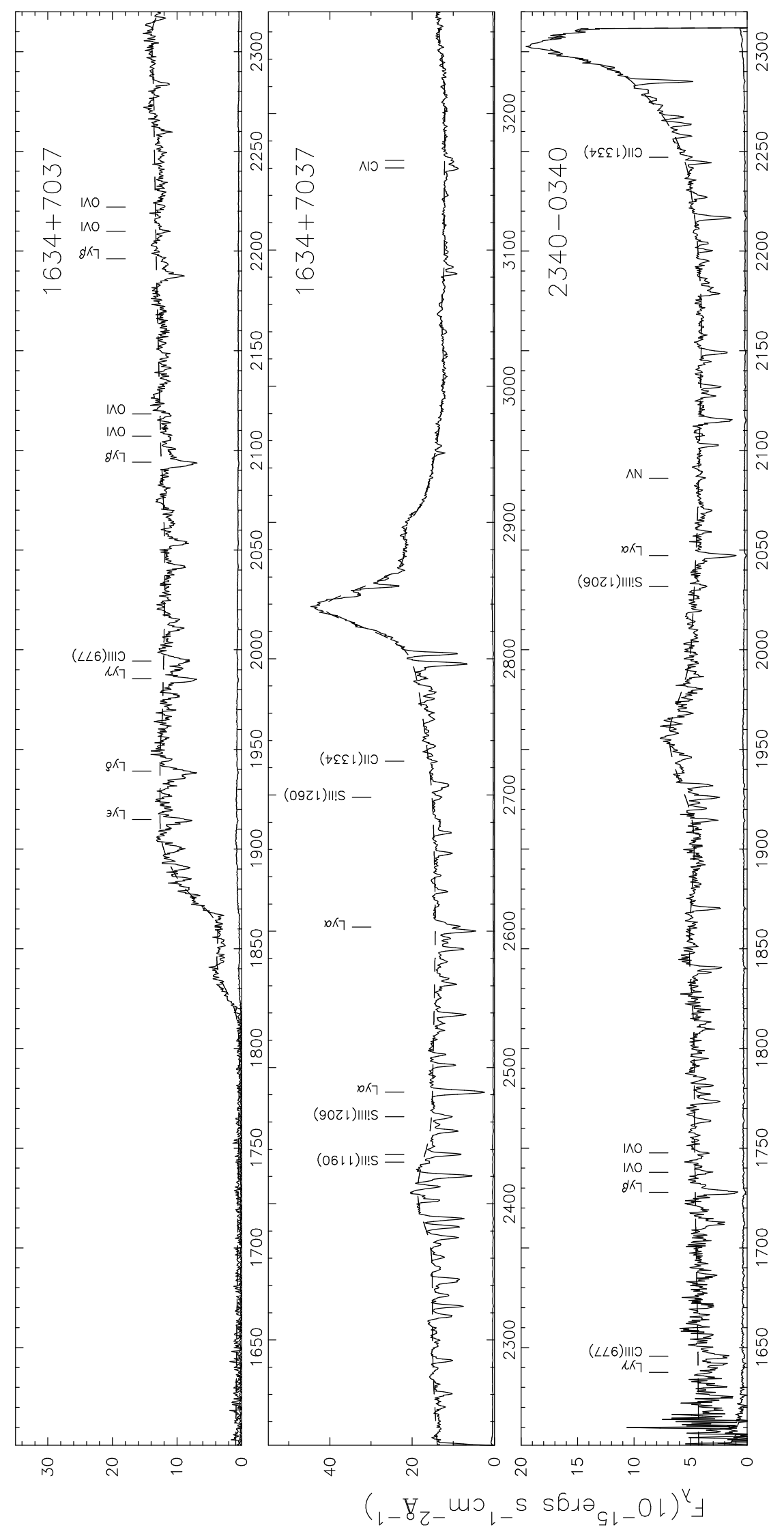

$\underset{1}{0<}$
$\frac{1}{ \pm}$
$\frac{1}{1}$
$\frac{1}{1}$
$\geq$
$\frac{0}{3}$ 


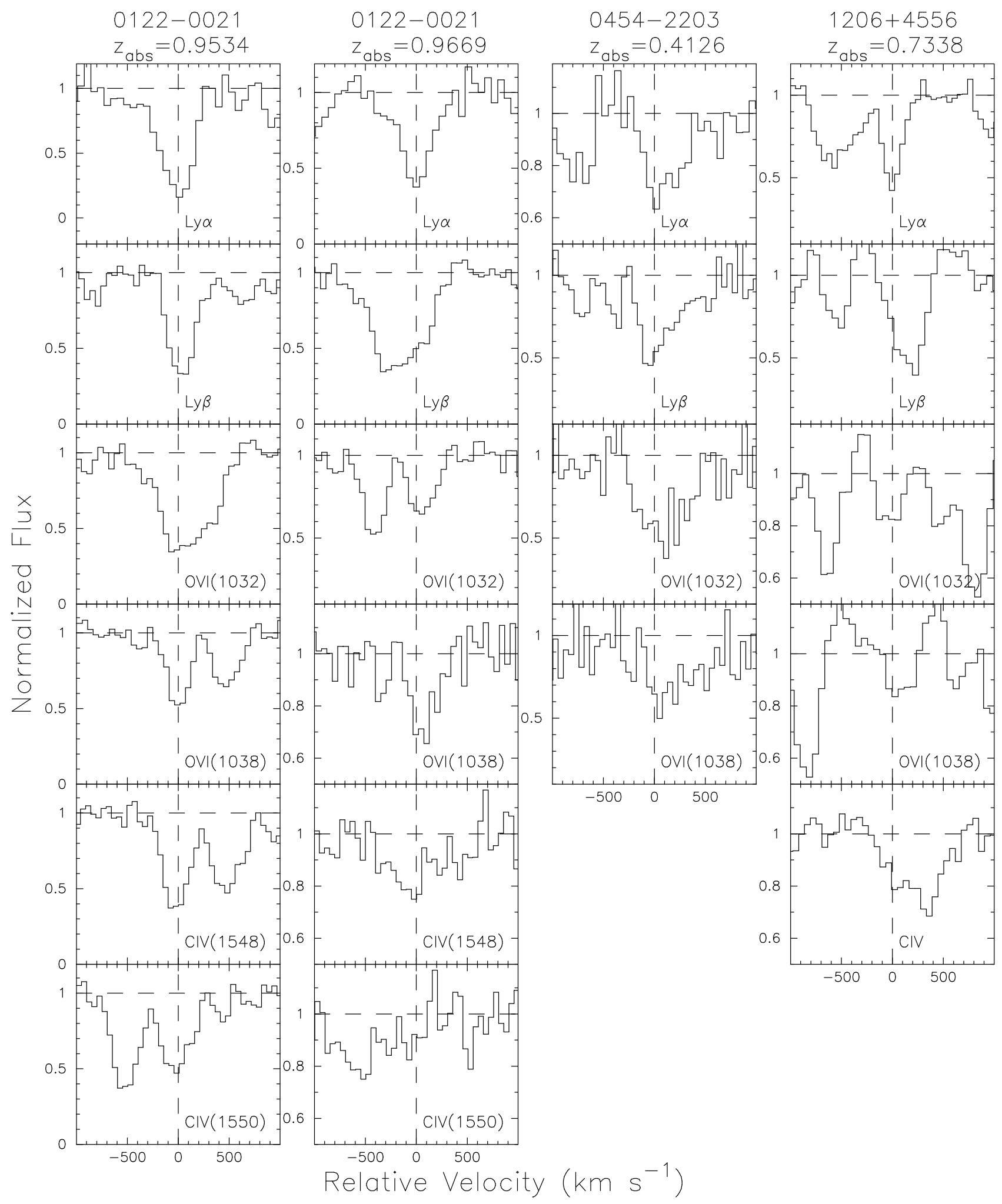




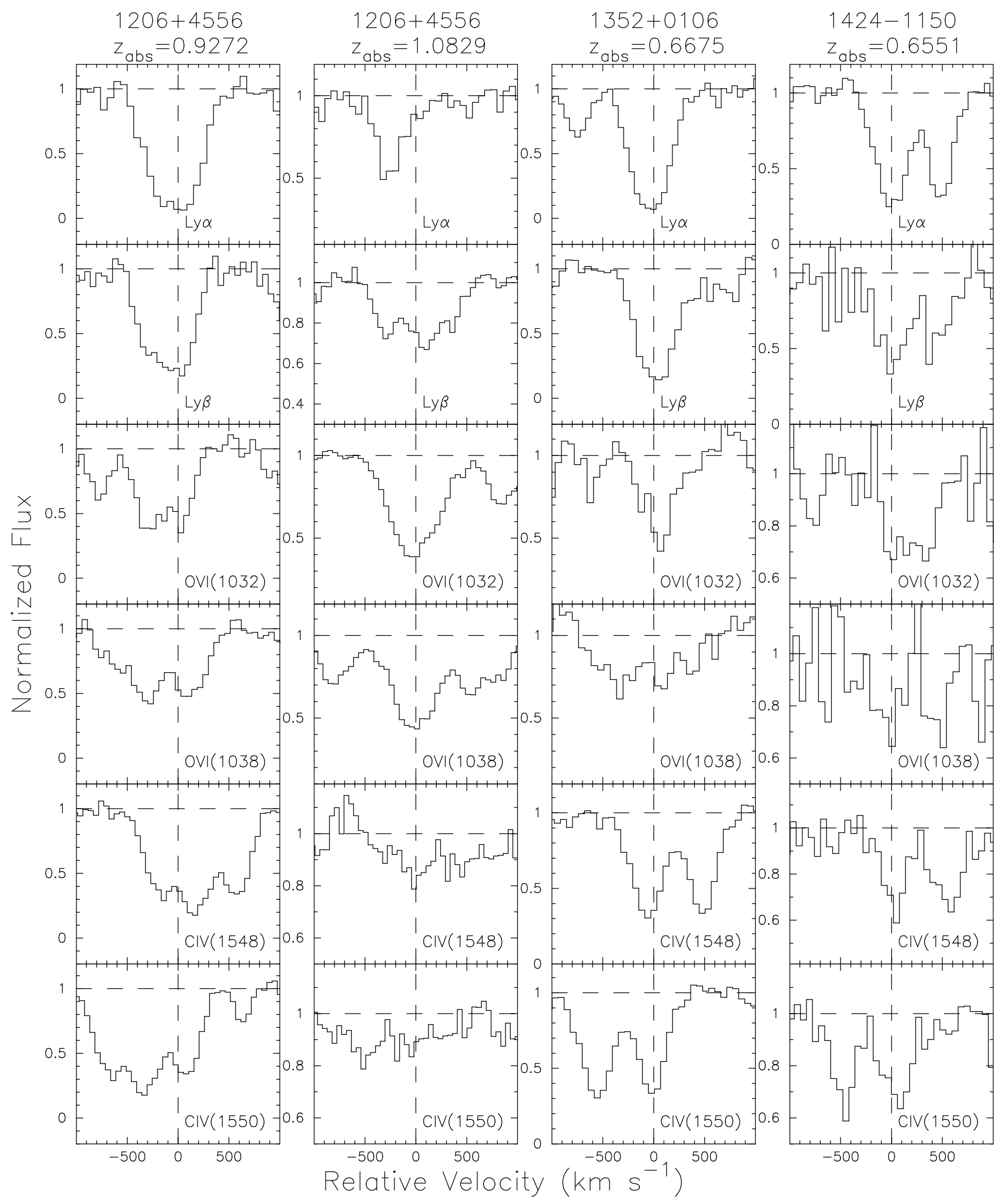




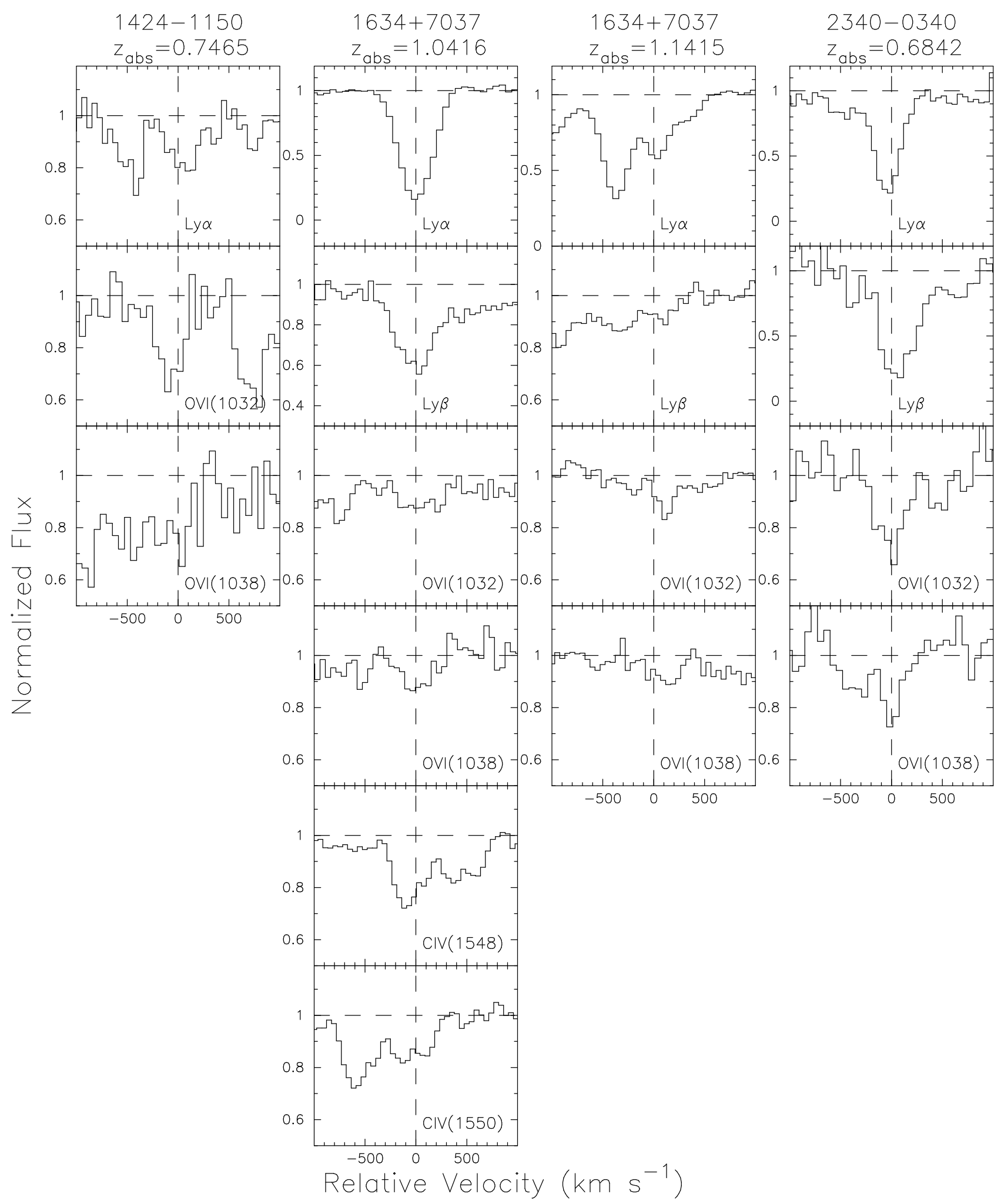




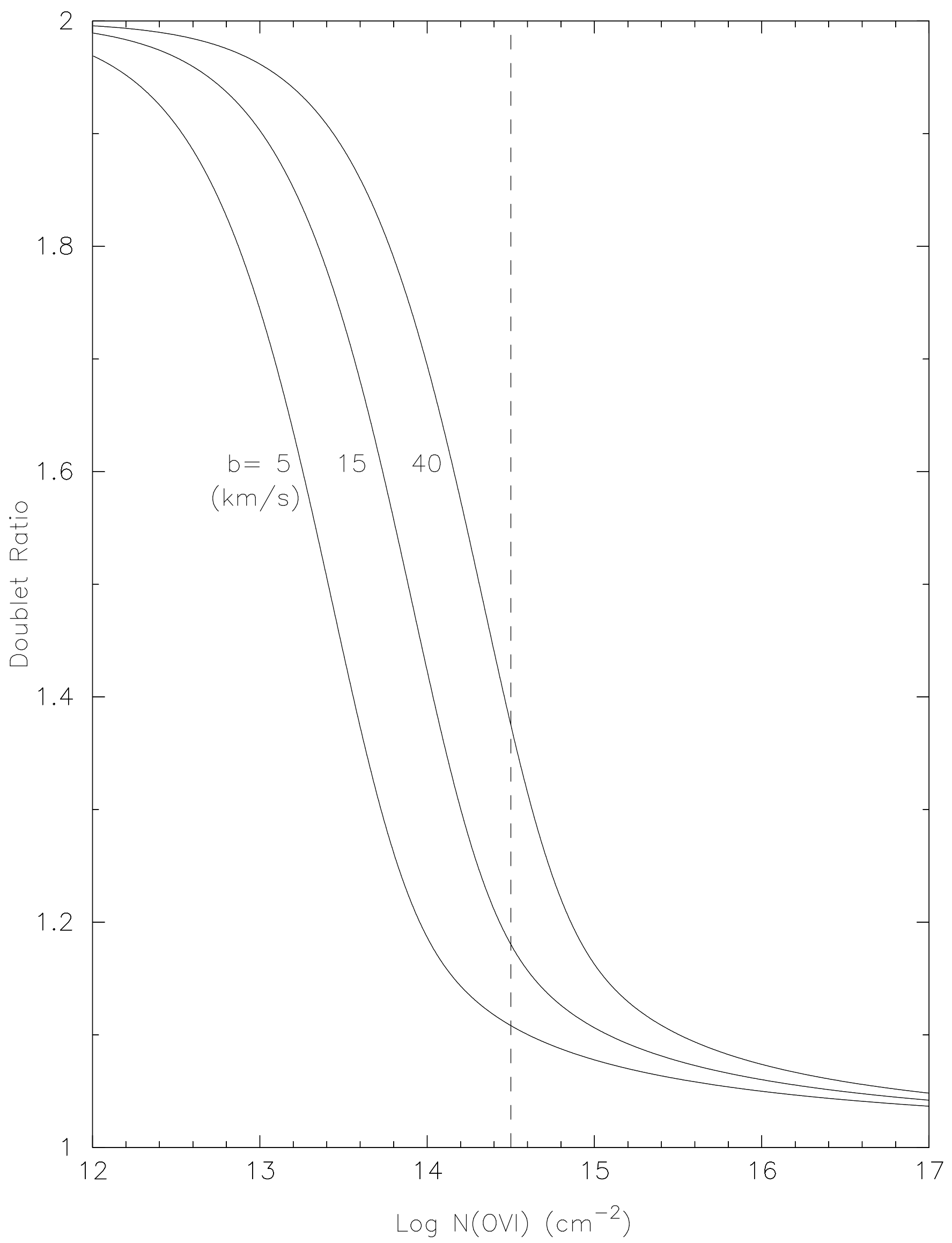




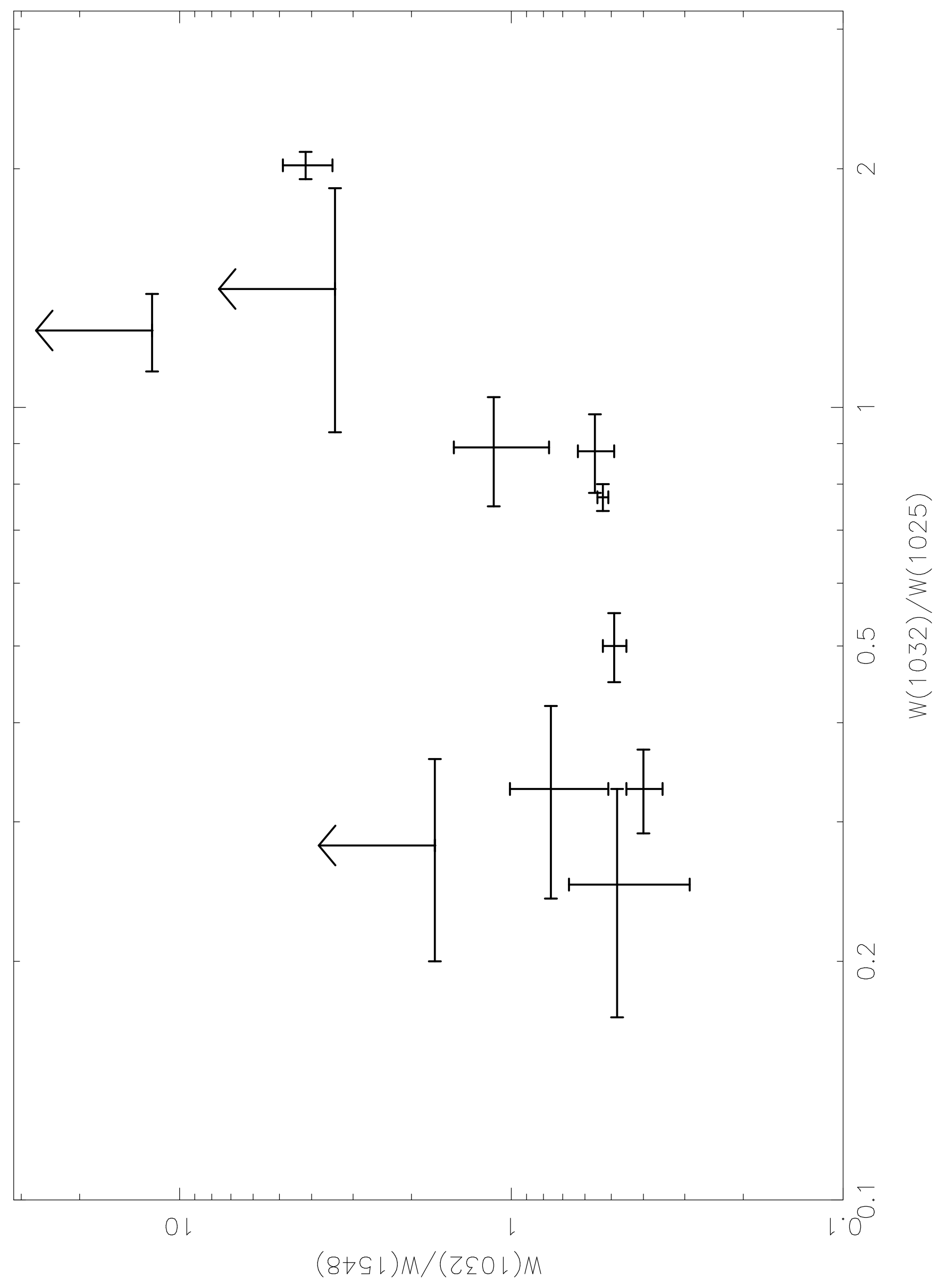

